This item is the archived peer-reviewed author-version of:

\title{
Fusion of hyperspectral and LiDAR data for classification of cloud-shadow mixed remote sensed scene
}

\section{Reference:}

Luo Renbo, Liao Wenzhi, Zhang Hongyan, Zhang Liangpei, Scheunders Paul, Pi Youguo, Philips Wilfried.- Fusion of hyperspectral and LiDAR data for classification of cloud-shadow mixed remote sensed scene IEEE journal of selected topics in applied earth observation and remote sensing / IEEE geoscience and remote sensing society; IEEE committee on earth observations - ISSN 1939-1404 - 99(2017), p. 1-14

Full text (Publishers DOI): http://dx.doi.org/doi:10.1109/JSTARS.2017.2684085 


\title{
Fusion of Hyperspectral and LiDAR Data for Classification of Cloud-Shadow Mixed Remote Sensed Scene
}

\author{
Renbo Luo, Student Member, IEEE, Wenzhi Liao, Senior Member, IEEE, Hongyan Zhang, Senior Member, IEEE, \\ Liangpei Zhang, Senior Member, IEEE, Paul Scheunders, Senior Member, IEEE, Youguo Pi, and Wilfried \\ Philips, Senior Member, IEEE,
}

\begin{abstract}
Recent advances in sensor design allow to gather more useful information about the Earth's surface. Examples are hyperspectral (HS) and Light Detection And Ranging (LiDAR) sensors. These however have limitations. HS data cannot distinguish different objects made from similar materials and highly suffers from cloud-shadow regions whereas LiDAR cannot separate distinct objects that are at the same altitude. For an increased classification performance, fusion of HS and LiDAR data recently attracted interest but remains challenging. In particular these methods suffer from a poor performance in cloud-shadow regions because of lack of correspondence with shadow-free regions and insufficient training data. In this paper, we propose a new framework to fuse HS and LiDAR data for classification of remote sensing scenes mixed with cloudshadow. We process the cloud-shadow and shadow-free regions separately, our main contribution is the development of a novel method to generate reliable training samples in the cloud-shadow regions. Classification is performed separately in the shadow-free (classifier is trained by the available training samples) and cloudshadow regions (classifier is trained by our generated training samples) by integrating spectral (i.e. original HS image), spatial (morphological features computed on HS image) and elevation (morphological features computed on LiDAR) features. The final classification map is obtained by fusing the results of the shadowfree and cloud-shadow regions. Experimental results on a real HS and LiDAR dataset demonstrate the effectiveness of the proposed method, as the proposed framework improves the overall classification accuracy with $4 \%$ for whole scene and $10 \%$ for shadow-free regions over the other methods.
\end{abstract}

Manuscript received xx; revised xx; accepted xx. This work was supported by FWO project G037115N: Data fusion for image analysis in remote sensing, the China Scholarship Council, and Open Fund of State Key Laboratory of Remote Sensing Science (Grant No.: OFSLRSS201610).

R. Luo is with the School of Automation Science and Engineering, South China University of Technology, 510640 Guangzhou, China, and also with the Department of Telecommunications and Information Processing, Ghent University, 9000 Ghent, Belgium (e-mail: renbo.luo@ugent.be).

W. Liao is with the Department of Telecommunications and Information Processing, Ghent University, Sint-Pietersnieuwstraat 41, 9000 Ghent, Belgium, and also with the State Key Laboratory of Remote Sensing Science, Institute of Remote Sensing and Digital Earth, Chinese Academy of Sciences. Beijing 100101, China (e-mail: wenzhi.liao@ugent.be).

H. Zhang and L. Zhang are with the State Key Laboratory of Information Engineering in Surveying, Mapping, and Remote Sensing and the Collaborative Innovation Center of Geospatial Technology, Wuhan University, Wuhan 430079, China (e-mail: \{zhanghongyan, zlp62\}@whu.edu.cn).

P. Scheunders is with Vision Laboratory, University of Antwerp, 2610 Antwerp, Belgium (e-mail: paul.scheunders@uantwerpen.be).

Y. Pi is with the School of Automation Science and Engineering, South China University of Technology, 510640 Guangzhou, China (e-mail: auygpi@ scut.edu.cn).

W. Philips is with the Department of Telecommunications and Information Processing, Ghent University, 9000 Ghent, Belgium (e-mail: wilfried.philips@ugent.be).
Index Terms-Hyperspectral image, LiDAR, classification, feature fusion, cloud-shadow

\section{INTRODUCTION}

$\mathbf{R}$ ECENT advances in sensor technology allow us to measure different aspects of the objects on the Earth's surface, e.g. the spectral radiance using hyperspectral (HS) images, and height information using Light Detection And Ranging (LiDAR) data [1]. Nowadays, HS images of both high spatial and spectral resolutions are available and can provide valuable spectral information for land use/cover applications [2]. However, their use is still limited in very complex scenes in which many objects are made of similar materials (e.g. roofs, parking lots and roads) [3], [4]. Moreover, optical in nature, hyperspectral sensors suffer from cloudy weather conditions. On the other hand, LiDAR image provides complementary information related to the size, structure and elevation of different objects [5], but fails to discriminate between different objects that are similar in altitude while quite different in nature (e.g. grass field and swimming pool). Therefore, using a single data source (either HS or LiDAR data) alone might not be sufficient to obtain reliable classification results.

Due to an increased availability of hyperspectral and LiDAR data from overlapping areas, the fusion of HS and LiDAR data has recently been explored intensively. In [6], Gu et al. proposed a multiple-kernel learning (MKL) model to integrate heterogeneous features from HS and LiDAR data for urban area classification. Elakshe et al. [7] explored the fusion of HS and LiDAR data for coastal mapping by using hyperspectral imagery to discriminate between road and water pixels, and LiDAR image to detect and create a vector layer of building polygons. Dalponte et al. [8] investigated the joint use of HS and LiDAR data for the classification of complex forest areas. Yokoya et al. [9] fused HS and LiDAR data for landscape visual quality assessment and enabled the prediction of landscape quality from any viewpoint using large-scale remote sensing observations. In [10], classification of eight common savanna tree species was performed by fusing HS and LiDAR data with an automated Random Forest modelling approach. Shimoni et al. proposed a score-level fusion approach to detect stationary vehicles under shadows in [11], where detection scores from both HS and LiDAR data are derived separately and combined with a simple sum rule. It is clear that the combination of 


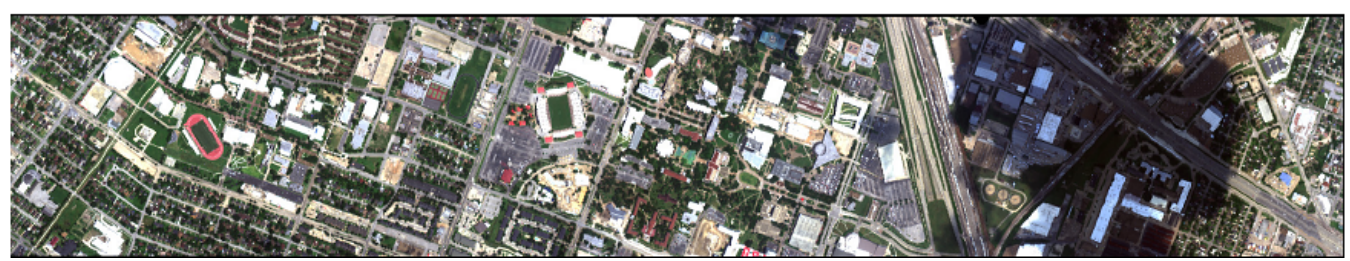

(a)

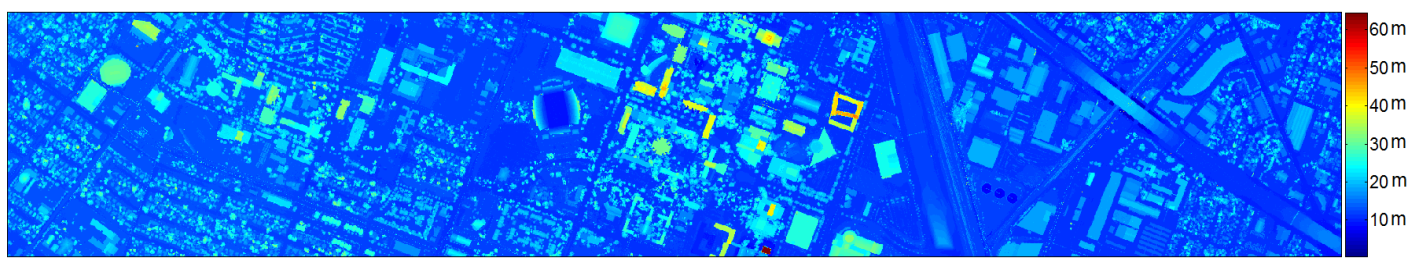

(b)

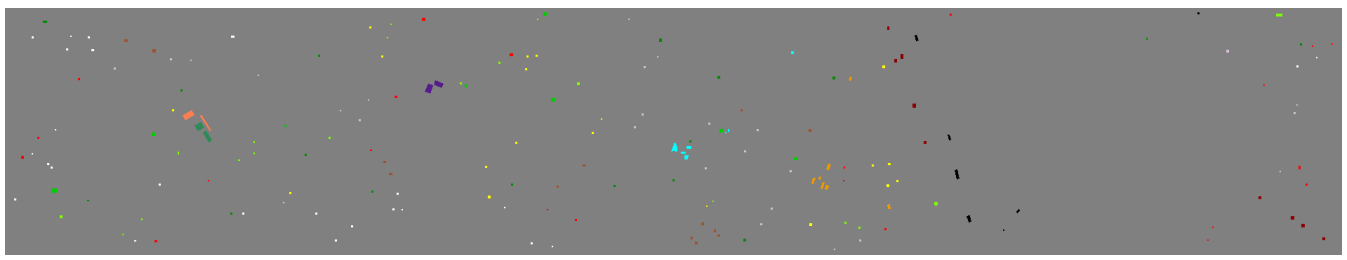

(c)

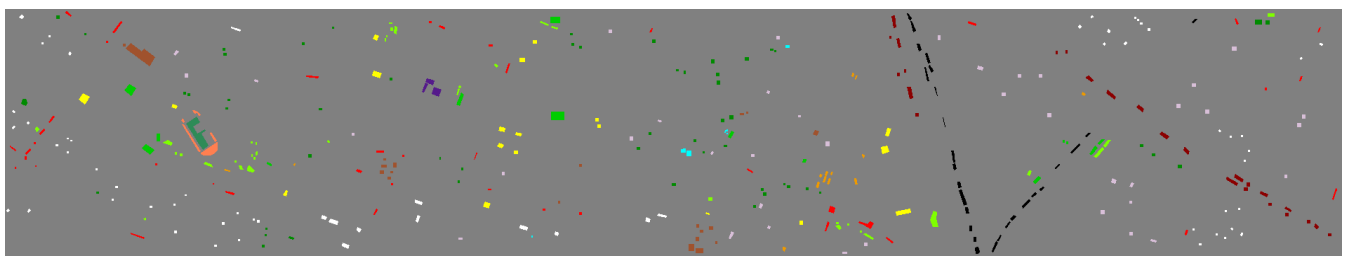

(d)

Fig. 1: (a) False RGB image of HS data; (b) LiDAR image; (c) available training samples; (d) testing samples.

HS and LiDAR data can contribute to a more comprehensive interpretation of ground objects [6]- [13].

As the footprint of one object often contains more than one pixel and thus a high spatial correlation is expected between neighboring pixels, many approaches [14]-[16] incorporated spatial information to improve the fusion process of HS and LiDAR data and address the salt and pepper phenomenon in classification. In [12], Pedergnana et al. applied morphological attribute profiles (MAPs) [17] to model information from both HS and LiDAR data, and fused multiple feature sources in a stacked architecture. Recently, Khodadadzadeh et al. [18] developed a new strategy to fuse HS and LiDAR data by stacking multiple types of features (spatial and spectral features from HS, elevation features from LiDAR). The methods mentioned above have demonstrated that combining spectral, spatial and elevation features further boosts the accuracy of landcover classification maps. However, stacking the high dimensional spectral and morphological features directly may lead to the curse of dimensionality problem [19] and excessive computation time.

In 2013, the Data Fusion Technical Committee of the IEEE Geoscience and Remote Sensing Society (GRSS) organized a contest involving two types of data sources: a cloud-shadow hyperspectral image and a LiDAR derived digital surface model (DSM) [20], see Fig 1. The competition was established to stimulate the development of advanced methods to fuse
HS and LiDAR data for classification [21]. More than 900 researchers from universities, national labs, space agencies and corporations across the globe registered to the contest and provided solutions. In particular, a graph-based fusion method [21] and its generalized version [22] were proposed to couple dimensionality reduction and feature fusion of the spectral information (of the original HS image) and MAPs (built on both HS and LiDAR data). Debes et al. [21] proposed a two-stream classification framework which combined the HS and LiDAR data by a parallel process that involves both unsupervised and supervised classification. In very recent work, Ghamisi et al. [25] fused hyperspectral and LiDAR data by using extinction profiles and deep convolutional neural networks and achieved improved classification results.

It is noteworthy that a large cloud shadow was present during the acquisition of the HS image. As cloud shadows blur most of the spectral information, they prevent accurate landcover mapping [26]. Moreover, as the clouds emerge and move irregularly and unpredictably, it is very difficult to label training samples and acquire remote sensing images at the same time, and to prepare two distinct sets of training samples for shadow-free and cloud-shadow regions in a remote sensing scene. Typically, most of the pixels in the cloud-shadow regions will be misclassified when only using training samples selected from shadow-free regions, as the spectral radiance information of samples located within and out of the cloud- 

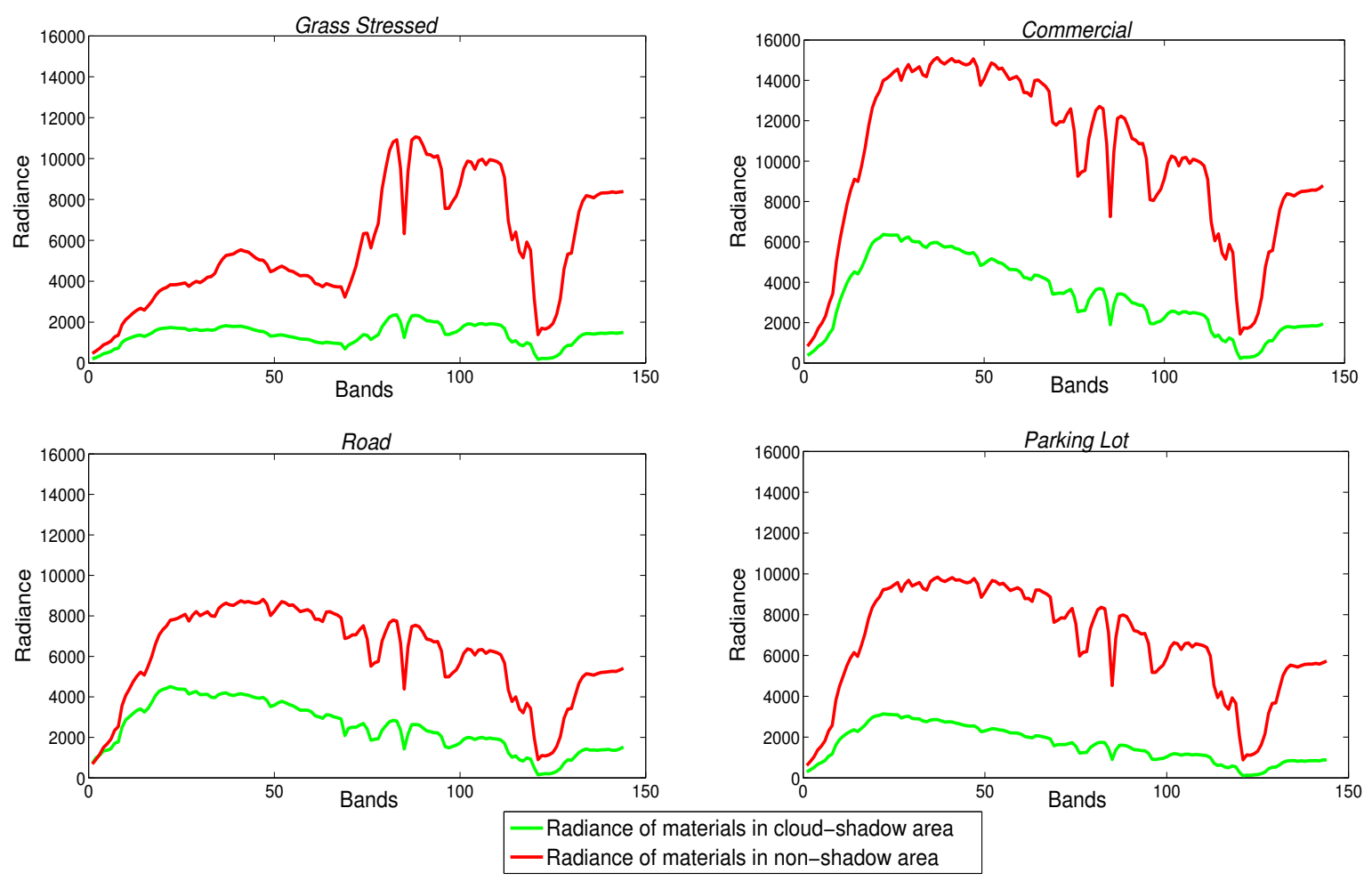

Fig. 2: Radiance (at-sensor spectral radiance units (SRU): $\mu \mathrm{W} \cdot \mathrm{cm}^{-2} \cdot \mathrm{sr}^{-1} \cdot \mathrm{nm}^{-1}$ ) of different materials.

shadow are totally different, see Fig. 2. For example, even though all the available training samples located in shadowfree regions were used to train the classifiers in [18]-[24], the classification performances of the cloud-shadow regions were not satisfactory.

In this paper, we propose a novel framework to fuse HS and LiDAR data for classification of remote sensing scenes mixed with cloud-shadow. The proposed method performs classification separately on the cloud-shadow and shadow-free regions. We solve the problem of missing training samples in the cloud-shadow regions by developing a method to generate reliable training samples. This method is based on the assumption that in the cloud-shadow regions different feature sources share similar intra-cluster distance relations, and that elevation features are more reliable than spectral and/or spatial features. We then classify the shadow-free (using the available training samples) and cloud-shadow regions (using the generated training samples) separately by integrating spectral, spatial and elevation features, obtained by exploiting attribute profiles [27]. The final classification map is produced by decision fusion of the obtained cloud-shadow and shadow-free maps.

We can also interpret our proposed framework from the viewpoint of domain adaptation [28]. The shadow-free region can be seen as the source domain, whereas the cloud-shadow region can be seen as a target domain. The labeled training set is only available for the source domain. According to the condition that the source and target domains share the same set of classes and elevation features (LiDAR), we make use of the information from the source domain to select training samples for the classification of the target domain.

The remainder of this paper is organized as follows: section III describes the proposed framework, with a detailed description of every part of the proposed method. The experimental results on real urban cloud-shadow HS and LiDAR data are presented and discussed in Section III. Finally, the conclusions of the paper are drawn in Section IV.

\section{Proposed Framework}

Clouds heavily distort the sun's reflectance and information analysis based on such distorted optical images is not always reliable. Meanwhile, collection of training data is preferably done on the ground, and since the clouds emerge and move irregularly and unpredictably, it is very difficult to obtain remote sensing images and label training samples from both cloud-shadow and shadow-free regions at the same time. Moreover, users prefer to select training samples from shadowfree regions for better visualization and interpretation. For example in Fig. 1c, all training samples were collected from shadow-free regions. When classifying a remote sensing scene with a classifier, trained on a shadow-free training set only, the results on the cloud-shadow regions will be very poor, the main reason being that objects made of the same material have different spectral signatures in cloud-shadow and shadow-free regions (Fig. 2). However, it is important to notice that within the cloud-shadow regions, objects made of different materials have different spectral signatures (Fig. 2), indicating that these regions still contain sufficient distinctive information. Some notations used throughout this paper are summarized in Table I) 
TABLE I: Some notations used in this paper.

\begin{tabular}{|c|c|}
\hline Notations & Description \\
\hline HS image & raw data cube: $M \times N \times D, D$ is the number of the spectral bands \\
\hline EMAPs $_{h s i}$ & attribute profiles generated from HS image: $M \times N \times D_{1}, D_{1}$ is the dimension of EMAPs ${ }_{h s i}$ \\
\hline $\mathrm{MAPs}_{l i d}$ & attribute profiles generated from LiDAR image: $M \times N \times D_{2}, D_{2}$ is the dimension of $\mathrm{MAPs}_{l i d}$ \\
\hline$n$ & number of labeled training samples \\
\hline$C$ & number of classes \\
\hline$y_{i}$ & label of $i$ th training sample \\
\hline$y_{i}^{\prime}$ & label of $i$ th sample in Maplid \\
\hline $\mathbf{x}_{i}^{\text {spe }}$ & $i$ th sample in HS image, $\mathbf{x}_{i}^{\text {spe }} \in \mathbb{R}^{D}$ \\
\hline $\mathbf{x}_{i}^{s p a}$ & $i$ th sample in $\mathrm{EMAPs}_{h s i}, \mathbf{x}_{i}^{s p a} \in \mathbb{R}^{D_{1}}$ \\
\hline $\begin{array}{c}\mathbf{x}_{i}^{S t a} \\
d\end{array}$ & $\begin{array}{l}i \text { th sample in the profile by stacking HS image and EMAPs }{ }_{h s i}, \mathbf{x}_{i}^{S t a}=\left\{\mathbf{x}_{i}^{\text {spe }} ; \mathbf{x}_{i}^{\text {spa }}\right\} \in \mathbb{R}^{D+D_{1}} \\
\text { the number of extracted features from each data source }\end{array}$ \\
\hline Fspe & spectral features extracted from HS image: $M \times N \times d$ \\
\hline Fspa & spatial features extracted from EMAPs ${ }_{h s i}: M \times N \times d$ \\
\hline Flid & elevation features extracted from $\mathrm{MAPs}_{l i d}: M \times N \times d$ \\
\hline $\mathbf{G}=\left\{g_{i j}\right\}$ & cloud-shadow mask \\
\hline $\mathbf{X}_{c(k)}^{\prime}$ & co-training samples for class $c$ in $k$ th iteration \\
\hline$n_{c}^{k}$ & number of samples in $\mathbf{X}_{c(k)}^{\prime}$ \\
\hline $\mathbf{m}_{c(k)}^{\text {spe }}$ & center of $\mathbf{X}_{c(k-1)}^{\prime}$ in spectral feature space, $\mathbf{m}_{c(k)}^{\text {spe }} \in \mathbb{R}^{D}$ \\
\hline $\mathbf{m}_{c(k)}^{s p a}$ & center of $\mathbf{X}_{c(k-1)}^{\prime}$ in spatial feature space, $\mathbf{m}_{c(k)}^{s p a} \in \mathbb{R}^{D_{1}}$ \\
\hline
\end{tabular}

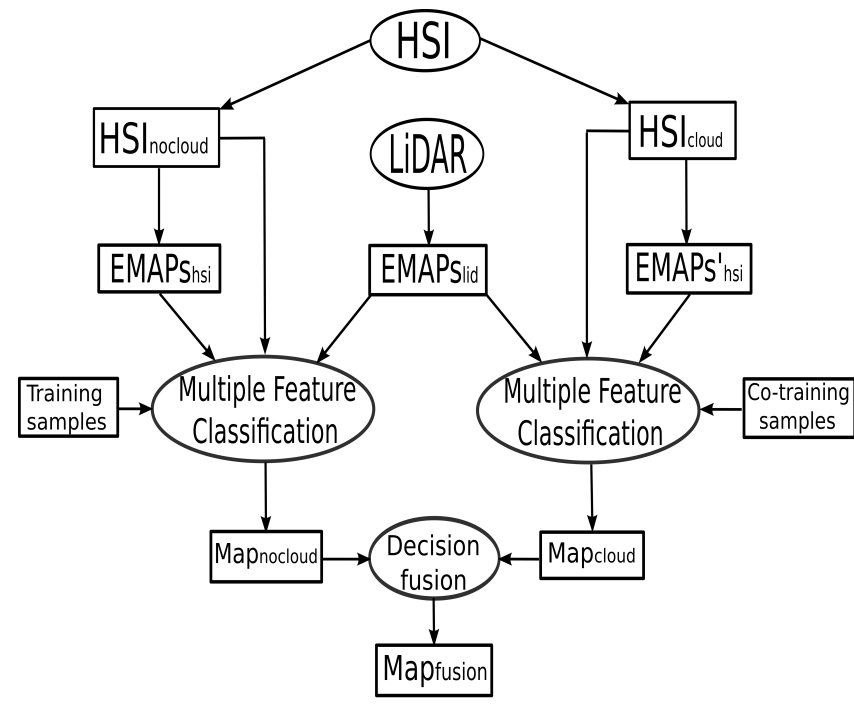

Fig. 3: Flowchart of the proposed framework, here HSI nocloud $_{1}$ and $\mathrm{HSI}_{\text {cloud }}$ denote the shadow-free and cloud-shadow regions of HS image, $\mathrm{EMAP}_{h s i}$ and $\mathrm{EMAP}_{h s i}$ mean morphological profiles extracted from hyperspecral and LiDAR data. 'Co-training samples' are generated training samples by our methods for cloud-shadow regions.

Therefore, we propose a novel framework for the classification of remote sensing scenes containing cloud shadows. In the proposed framework, as shown in Fig. 3, we first divide the HS image into two different parts: cloud-shadow $\left(\mathrm{HSI}_{\text {cloud }}\right)$ and shadow-free $\left(\mathrm{HSI}_{\text {nocloud }}\right)$ regions. EMAPs $\mathrm{E}_{h i}$ and MAPs sid $_{l i}$ denote the additional spatial and elevation information extracted from HS and LiDAR data by attribute profiles [27], respectively. For the classification of shadowfree regions $\mathrm{HSI}_{\text {nocloud }}$, we fuse multiple features using a similar framework as in [29]. To reduce the information redundancy, we first use feature extraction (FE) techniques to extract relevant information from each single feature source. Then we concatenate all extracted features together, and use these as input for a classifier to obtain the classification map of shadow-free regions Map $_{\text {nocloud. }}$ In order to generate a classification map of cloud-shadow regions Map $_{\text {cloud }}$, we propose a novel method to select new training samples from cloud-shadow regions, which will be detailed in the following subsection. Using these training samples, the same multiple feature classification method as with the shadow-free regions is applied. Last but not least, the final classification map of a cloud mixed remote sensing scene is obtained by fusing Map $_{\text {nocloud }}$ and Map Mloud. $_{\text {. }}$

\section{A. Morphological Attribute Profiles (MoAP)}

For the classification of very high-resolution remote sensing data, spatial information has been widely exploited [30]- [33]. To model the spatial information from HS and LiDAR data, Pesaresi et al. [30] build so-called morphological profiles (MP). As an extension of the concept of MP, attribute profiles (AP) [27] provide a multilevel characterization of an image by the sequential application of morphological attribute filters, which model different specifications of the structural information contained in the scene, such as length, area and shape of objects. In [31], [32], extended multi-attribute profiles (EMAPs) were developed to extract abundant spatial information in HS images and these profiles make an obvious contribution to the classification.

Attribute profiles (AP) generate a multi-level decomposition of the input image based on attribute filters, which can properly extract and model the spatial information of the adjacent pixels with progressively higher threshold values. Suppose $\lambda=\left\{\lambda_{1}, \lambda_{2}, \cdots, \lambda_{n}\right\}\left(\lambda_{i}<\lambda_{j}\right.$ with $\left.i<j\right)$ is a sequence of ordered predefined criteria, for a gray scale image $g$, then an AP of $g$ can be defined as:

$$
\mathbf{A P}(g)=\left\{\phi_{n}(g), \cdots, \phi_{1}(g), g, \varphi_{1}(g), \cdots, \varphi_{n}(g)\right\}
$$




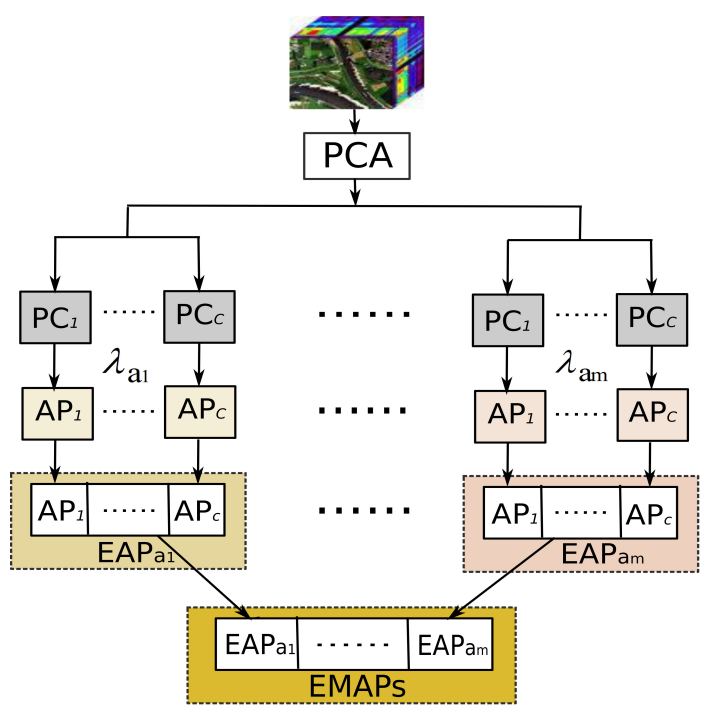

Fig. 4: General architecture of EMAPs, $\lambda_{a_{i}}$ demotes the predefined conditions of attribute $a_{i}$.

where $\phi_{i}$ and $\varphi_{i}$ denote the attribute thinning and thickening operations with reference values $\lambda_{i}$.

The above AP only works on a gray scale image. In order to extend the concept of the AP to HS image, one possible way is to perform a feature reduction approach (such as PCA) on the input data and then apply APs to the first principle components [32]. Let $P C_{i} i=\{1, \ldots, c\}$ demotes the first principle components of HS image, then extended-AP (EAP) can be mathematically given as:

$$
\mathbf{E A P}=\left\{\mathbf{A P}\left(P C_{1}\right), \mathbf{A P}\left(P C_{2}\right), \cdots, \mathbf{A P}\left(P C_{c}\right)\right\}
$$

The presented EAPs model the size and structure of different objects based on one attribute. If more attributes (e.g. area, diagonal of bounding box, length and standard deviation) are considered, extended multi-attribute profiles (EMAPs) can be denoted as:

$$
\mathbf{E M A P s}_{h s i}=\left\{\mathbf{E A P}_{a_{1}}, \overline{\mathbf{E A P}}_{a_{2}}, \cdots, \overline{\mathbf{E A P}}_{a_{m}}\right\}
$$

where $a_{i}$ is a generic attribute and $\overline{\mathbf{E A P}}=\mathbf{E A P}$ । $\left(P C_{1}, P C_{2}, \cdots, P C_{c}\right)$, deleting $P C s$ from $\mathrm{EAP}_{a_{i}}, i>1$ is necessary for avoiding redundancy since the original components $P C_{i}$ are present in each EAP. Fig 4 shows the general architecture of EMAPs Esi $_{h i}$.

AP can also be applied to model elevation information from LiDAR image. An attribute thinning acts on bright objects (for LiDAR image, the bright regions are actually areas with high elevation, such as the top of a roof), while thickening act on dark (low height) objects. For example, an attribute thinning deletes bright objects that are smaller than the threshold $\lambda_{i}$. By computing a series of attributes, a complete attribute profile is built, carrying information about the elevation information of objects in the image. Suppose $L$ denotes LiDAR image, $L$ can be seen as a gray scale image where the value of a pixel denotes the altitude at that point. Then the AP of $L$ can be defined as:

$$
\mathbf{A P}(L)=\left\{\phi_{n}(L), \cdots, \phi_{1}(L), L, \varphi_{1}(L), \cdots, \varphi_{n}(L)\right\}
$$

As LiDAR-derived image has only single band, we use the term multi-attribute profiles (MAPs) when different types of APs are applied to the LiDAR image. Then the MAPs from LiDAR image (MAPs $\mathbf{M}_{l i d}$ ) can be expressed as:

$$
\mathbf{M A P s}_{l i d}=\left\{\mathbf{A P}_{a_{1}}(L), \mathbf{A} \mathbf{P}_{a_{2}}(L), \cdots, \mathbf{A} \mathbf{P}_{a_{m}}(L)\right\}
$$

where $a_{i}, i=\{1, \cdots, m\}$ denotes different types of attributes.

Fig. 5 shows some of the obtained APs on the HS and LiDAR image. The objects of the hyperspectral image under cloud shadow appear to be darker. Moreover, many object$s$ (even in different categories) exhibit similar intensities (Fig. 5a. APs of LiDAR image are clearly less influenced by the cloud (Fig. 5b), small objects disappear as the scale increases. With EMAP, additional spatial and elevation can be extracted.

\section{B. Multiple Features Classification}

From HS and LiDAR data, three types of feature sources can be obtained: spectral values from the original spectrum of the HS image, EMAPs $s_{h i}$ from the HS image and MAPs ${ }_{l i d}$ from the LiDAR image, all of them having high dimensionality. If these features would be fused using a stacked structure, the dimensionality of this stacked vector will be very large, thus leading to the problem of the curse of dimensionality. Moreover, the stacked vector will contain redundancy and noisy. Therefore, it is necessary to use feature extraction (FE) methods to reduce the dimensionality of the spectral features, the EMAPs EMsi $_{\text {and }}$ MAPs ${ }_{l i d}$ before fusing the extracted lowdimensional features together for classification. Here nonparametric weighted feature extraction (NWFE) [34] is chosen, as it is proven to be efficient to extract discriminative features for classification of HS image [32].

Fig. 6a shows the proposed multiple feature classification strategy. First, the original HS data is transformed by FE to obtain a reduced set of effective spectral features ( $F$ spe) that contains the spectral information of the HS data. In parallel, the HS image is transformed by PCA, and the first few important PCs that correspond to $99 \%$ of the cumulative variance are used to construct the EMAPs $_{h s i}$. If there are $c$ $P C s$, each AP is composed of $n$ thickening and $n$ thinning transformations of the corresponding $P C$ for each attribute and the number of attributes is $m$, then there are in total $c \times(m \times(2 n)+1)$ features in the EMAPs. In order to reduce redundancy and noise, avoid the curse of dimensionality and save processing time, FE is applied to extract an effective feature set $(F s p a)$ from EMAPs ${ }_{h s i}$ before classification. On the LiDAR image, exactly the same is done to extract an ef-

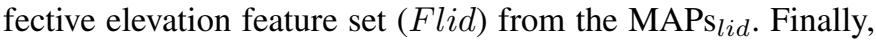
the obtained Fspe, Fspa and Flid are concatenated into one stacked vector Ffusion.

Another strategy would be to make up a large stacked vector from the spectral features, the spatial profiles of EMAPs ${ }_{h s i}$

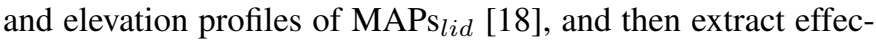
tive features from this large stacked vector (Fig. 6b). In that case however, since the different feature sources have different distributions, the information is not equally represented in the stacked vector, and some important features may get lost or 

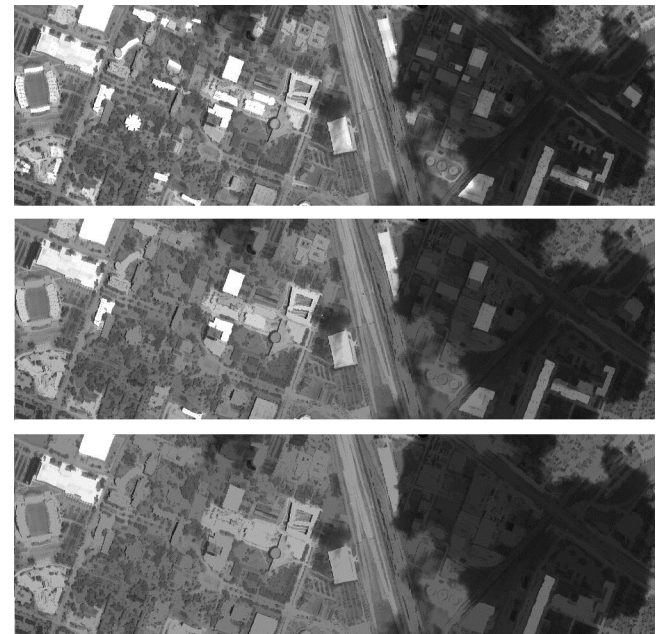

(a)

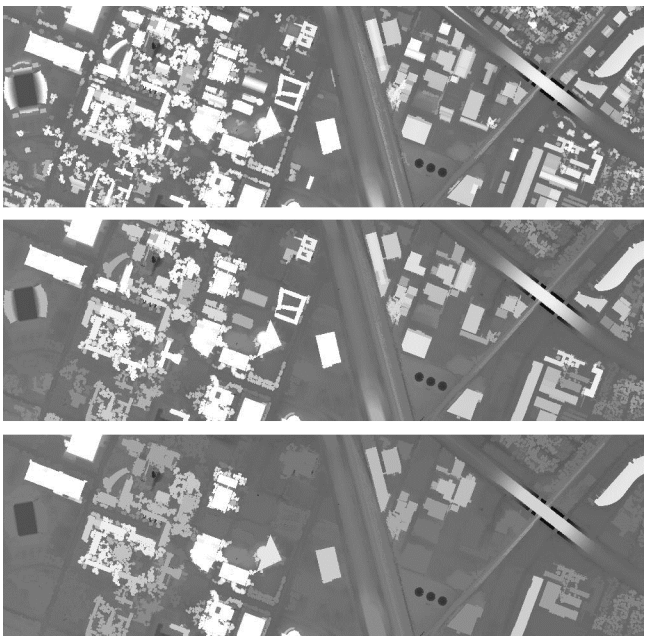

(b)

Fig. 5: Attribute thinning with "area" attribute. From up to down, the area size was set to 200, 500, and 1000 respectively. (a) APs of hyperspectral image with first PC; (b) APs of LiDAR image.

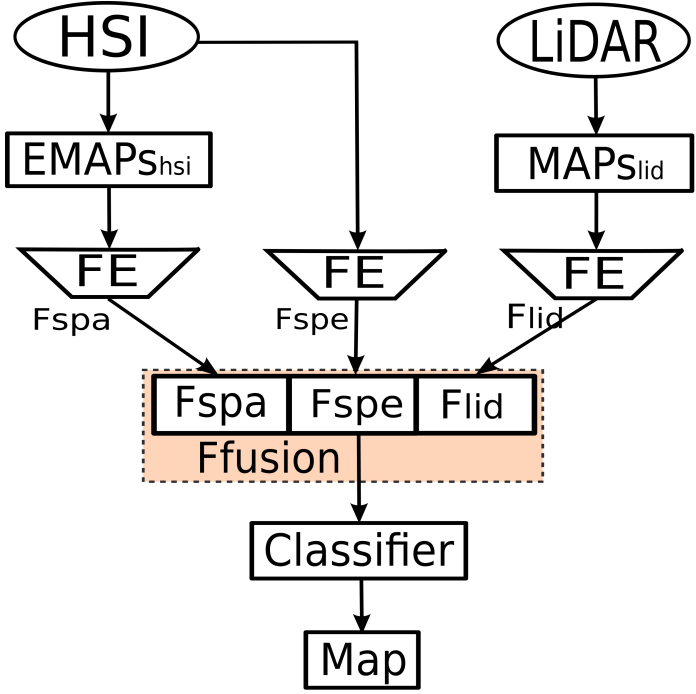

(a)

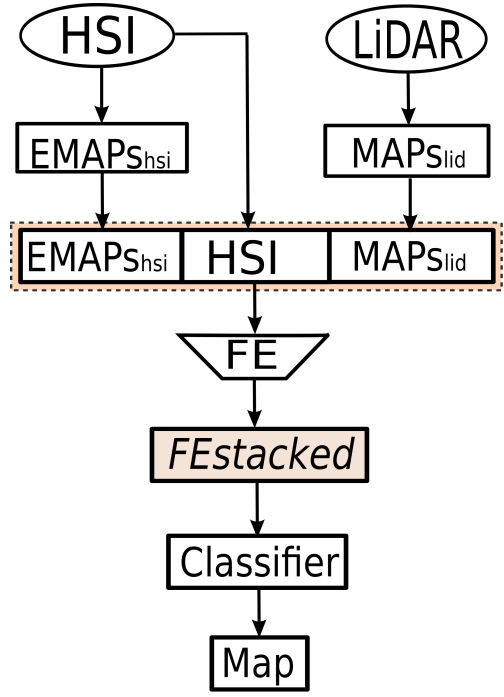

(b)

Fig. 6: (a) Proposed multiple feature classification; (b) Multiple feature classification proposed in [18].

mixed if we project stacked features from different sources into a low-dimensional feature space together. We verified experimentally that the first strategy is the more effective one.

\section{Cloud-shadow Detection}

Cloud shadow cannot be always avoided during the acquisition of optical remote sensing data. The presence of cloud and shadow complicates the analysis of remote sensing data, leading e.g. to false detection of land cover change [26], biased estimation of Normalized Difference Vegetation Index (NDVI) values, and mistakes in classification tasks. Therefore, the detection of cloud shadow is an initial and important step [35]. Actually, many approaches have been developed to detect cloud shadow, such as geometry-based methods [36] and the Fmask algorithm [37], [38].

Since cloud-shadow detection is not the primary goal of this work, we will apply a simple method based on area attribute filters [31] to detect the big cloud-shadow region, because in our specific case study area, the area of the cloud-shadow is much larger and darker than other ground objects. By increasing the thresholds of the area attribute, more and more bright objects are filtered out, leaving finally the largest dark cloud shadow region (Fig. 7b). The cloud-shadow mask is then obtained by binarizing the result (Fig. 7c). In fact, there is a very small cloud-shadow region present at the top center-right of the image. We just choose the large main cloud-shadow as an example here, as all shadowed testing samples are located in this large cloud-shadow region. Denote $\mathbf{G}=\left\{g_{i j}\right\}$ as the cloud-shadow mask, with pixel values $g_{i j}=0$ in the cloudshadow region and $g_{i j}=1$ in the shadow-free region.

\section{Co-training Samples Selection}

In this section, we describe a new method to select and label a separate training set (called co-training samples) for the cloud-shadow regions. Since LiDAR image are not influenced by clouds, our proposed method uses single elevation information (i.e., Flid, see section II-B to obtain an initial classification map $\left(\mathrm{Map}_{\text {lid }}\right)$. However, single elevation 


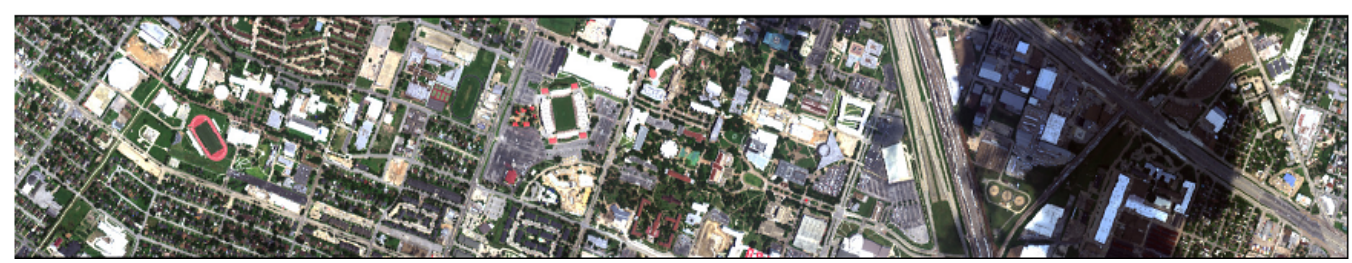

(a)

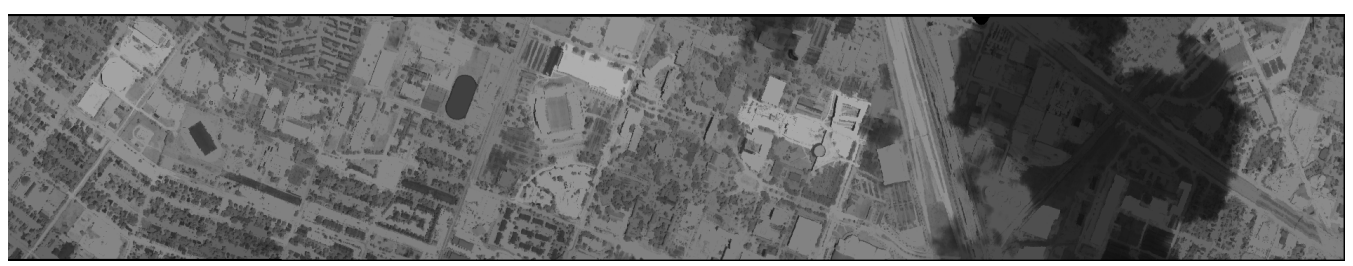

(b)

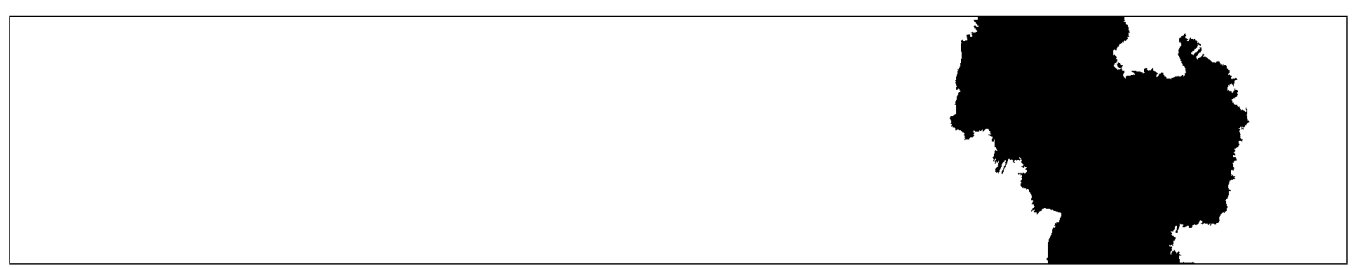

Fig. 7: (a) False RGB image of HS data; (b) Area attribute thinning with area size 3000 of HS data; (c) Extracted cloud-shadow map.

information from LiDAR image is not sufficient for a reliable classification, as many man-made objects in urban areas are of similar height. Therefore, we combine the spectral and spatial information from the HS image with Map lid $_{\text {tid }}$ to select new cotraining samples from cloud-shadow regions in the following way(Fig 8a):

Suppose $\mathbf{X}^{S t a}=\left\{\mathbf{x}_{i}^{S t a}\right\}_{i=1}^{n}$ denote the set of samples, $\mathbf{x}_{i}^{S t a}=\left\{\mathbf{x}_{i}^{s p e} ; \mathbf{x}_{i}^{s p a}\right\}, \mathbf{Y}^{\prime}=\left\{y_{i}^{\prime}\right\}_{i=1}^{n}, \mathbf{x}_{i}^{s p e}$ and $\mathbf{x}_{i}^{s p a}$ denote the spectral information in the HS image and spatial information in EMAPs Shi $_{h i}$ of the $i$ th pixel respectively, $y_{i}^{\prime} \in\{1, \cdots, C\}$ denotes the label of pixel $i$ in the classification map (Maplid, see Fig $8 \mathrm{a}$ obtained by the LiDAR feature source.

In fact, multiple feature sources (i.e., original spectrum of HS image, EMAPs $s_{h i}$ from HS image and MAPs $\mathrm{MAd}_{\text {lid }}$ from LiDAR image) can be seen as information from different aspects for pixels. For two samples, if their information are similar from all aspects, we assume they belong to same class and share same labels. Let $\mathbf{X}_{c(1)}^{\prime}$ be a set of initial selected cotraining samples which belong to class $c$. $\mathbf{X}_{c(1)}^{\prime}$ can be obtained from the following criterion:

$$
\begin{aligned}
\mathbf{X}_{c(1)}^{\prime}=\left\{\mathbf{x}_{i}^{S t a}\right. & : \mathbf{x}_{i}^{\text {spe }} \in \operatorname{knn}\left(\mathbf{m}_{c(1)}^{\text {spe }}\right) \cdots \\
\wedge \mathbf{x}_{i}^{\text {spa }} & \left.\in \operatorname{knn}\left(\mathbf{m}_{c(1)}^{s p a}\right)\right\}
\end{aligned}
$$

where

$$
\begin{aligned}
& \mathbf{m}_{c(1)}^{s p e}=\frac{1}{n_{c}^{0}} \sum_{i=1}^{n_{c}^{0}} \mathbf{x}_{i}^{s p e}, \text { where } y_{\mathrm{i}}^{\prime}=\mathrm{c} \\
& \mathbf{m}_{c(1)}^{s p a}=\frac{1}{n_{c}^{0}} \sum_{i=1}^{n_{c}^{0}} \mathbf{x}_{i}^{s p a}, \text { where } y_{\mathrm{i}}^{\prime}=\mathrm{c}
\end{aligned}
$$

" $\wedge$ " here means "and", $\mathbf{m}_{c(1)}^{s p e}$ and $\mathbf{m}_{c(1)}^{s p a}$ can be seen as the initial center of class $c$ in spectral feature space and spatial feature space respectively, $n_{c}^{0}$ is the number of initial selected co-training samples in class $c$ based on $\operatorname{Map}_{\text {lid }}, \operatorname{knn}\left(\mathbf{m}_{c(1)}\right)$ denotes the set of $k$-nearest neighbors of $\mathbf{m}_{c(1)}$. Here $k$ nearest neighbors are selected based on Euclidean distance, as Euclidean distance is simple and widely used in $k$-nearest neighbors searching. In this way, the selected co-training samples for each class have similar spectral, spatial and elevation information. For details see Fig $8 \mathrm{~b}$.

However, as it is only based on a single elevation feature source, the classification accuracies for some classes are relatively low in Maplid, leading to less accurate class center. As a result, the co-training samples selected based on equation (6) are not reliable. In order to solve this problem, we iteratively update the class centers, similar as in a mean shift algorithm. Suppose $\mathbf{X}_{c(k)}^{\prime}$ is a set of training samples belonging to class $c$, generated in the $k$ th iteration. This set can be obtained from the training set at the $(k-1)$ th iteration through the following criterion:

$$
\begin{aligned}
\mathbf{X}_{c(k)}^{\prime}=\left\{\mathbf{x}_{i}^{S t a}\right. & : \mathbf{x}_{i}^{s p e} \in \operatorname{knn}\left(\mathbf{m}_{c(k)}^{s p e}\right) \cdots \\
\wedge \mathbf{x}_{i}^{s p a} & \left.\in k n n\left(\mathbf{m}_{c(k)}^{s p a}\right)\right\}
\end{aligned}
$$

where

$$
\begin{aligned}
& \mathbf{m}_{c(k)}^{s p e}=\frac{1}{n_{c}^{(k-1)}} \sum_{i=1}^{n_{c}^{(k-1)}} \mathbf{x}_{i}^{s p e}, \text { where } \mathbf{x}_{\mathrm{i}}^{\mathrm{Sta}} \in \mathbf{X}_{\mathrm{c}(\mathrm{k}-1)}^{\prime} \\
& \mathbf{m}_{c(k)}^{s p a}=\frac{1}{n_{c}^{(k-1)}} \sum_{i=1}^{n_{c}^{(k-1)}} \mathbf{x}_{i}^{s p a}, \text { where } \mathbf{x}_{\mathrm{i}}^{\mathrm{Sta}} \in \mathbf{X}_{\mathrm{c}(\mathrm{k}-1)}^{\prime}
\end{aligned}
$$

$\mathbf{m}_{c(k)}^{s p e}$ and $\mathbf{m}_{c(k)}^{s p a}$ denote the centers of $\mathbf{X}_{c(k-1)}^{\prime}$ in spectral feature space and spatial feature space, respectively, $n_{c}^{(k-1)}$ is 


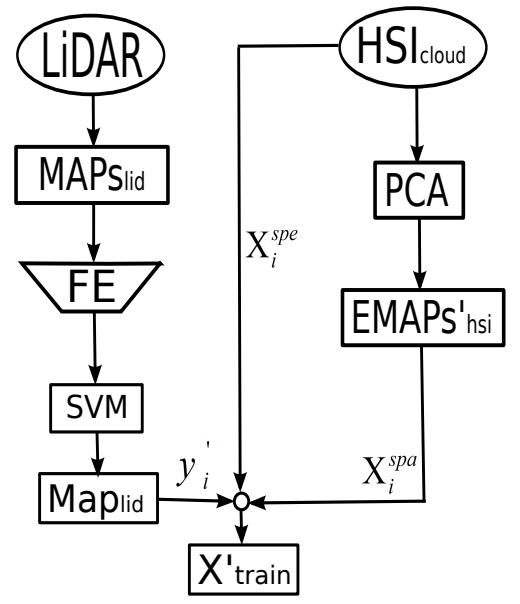

(a)

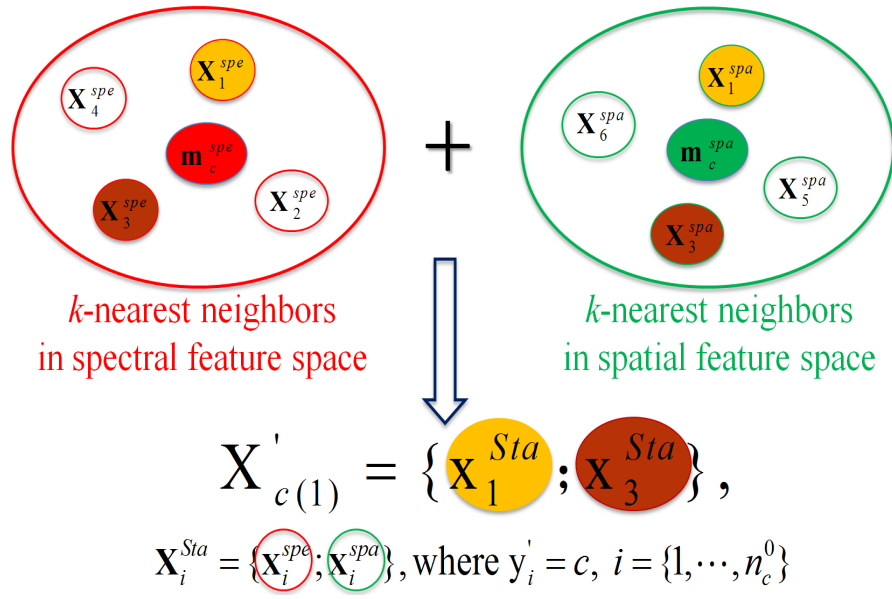

(b)

Fig. 8: (a) Co-training samples selection; $\mathbf{x}_{i}^{\text {spe }}$ and $\mathbf{x}_{i}^{\text {spa }}$ represent spectral radiance and EMAPs ${ }_{h s i}$ of the $i$ th pixel; (b) $\mathbf{x}_{i}^{S t a}=\left\{\mathbf{x}_{i}^{s p e} ; \mathbf{x}_{i}^{s p a}\right\}$; the pixel is labeled $c$ in $\operatorname{Map}_{\text {lid }}\left(y_{i}^{\prime}=c\right) ; \mathbf{m}_{c}^{s p e}$ and $\mathbf{m}_{c}^{s p a}$ are the centers of class $c$ in spectral and spatial feature space, here $\mathbf{x}_{1}^{S t a}$ and $\mathbf{x}_{3}^{S t a}$ are nearest neighbors of the center of class $c$, both in spectral and spatial feature space, and selected as candidate co-training samples.

the number of samples in $\mathbf{X}_{c(k-1)}^{\prime}$. The iteration procedure can be stopped by introducing 2 thresholds $\varepsilon^{s p e}$ and $\varepsilon^{s p a}$. When

$$
\left|\mathbf{m}_{c(k)}^{s p e}-\mathbf{m}_{c(k-1)}^{s p e}\right|<\varepsilon^{s p e} \wedge\left|\mathbf{m}_{c(k)}^{s p a}-\mathbf{m}_{c(k-1)}^{s p a}\right|<\varepsilon^{s p a}
$$

, the centers of co-training samples in each class are stable. We define the final co-training samples set as: $\mathbf{X}_{\text {train }}^{\prime}=$ $\left\{\mathbf{X}_{1(k)}^{\prime}, \mathbf{X}_{2(k)}^{\prime}, \cdots, \mathbf{X}_{c(k)}^{\prime}\right\}$. The algorithmic procedure of the proposed co-training samples selection method is formally stated in Algorithm 1

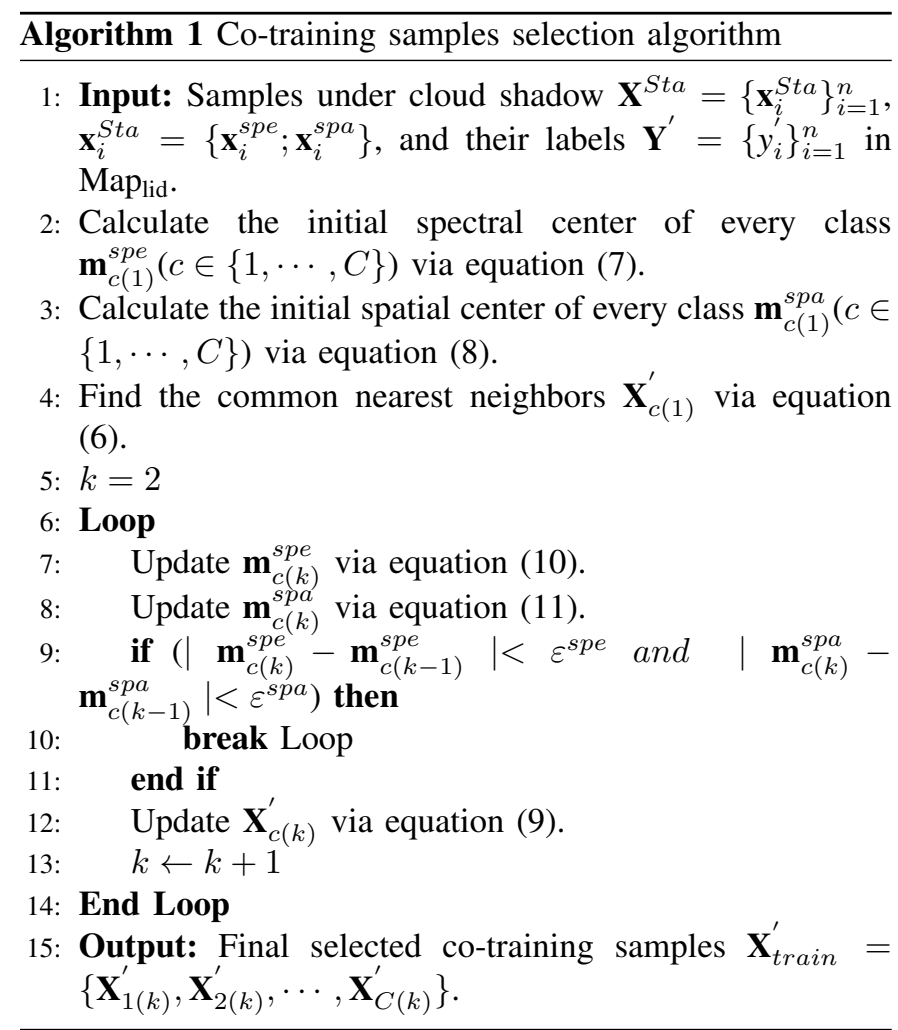

\section{E. Classification Map Fusion}

After obtaining the new co-training samples under cloudshadow regions, multiple features classification is applied in the same way as for cloud-free regions, the only difference being the way the training samples were obtained, where in the cloud-free regions, we use available training samples outside of the cloud mask, while in the cloud-shadow regions, we apply our proposed co-training samples selection procedure. The final classification map is obtained by fusion of the two maps: Map cloud $_{\text {and }}$ Map nocloud $_{\text {. }}$

$$
\text { Map }=g_{i, j} M_{\text {cloud }}+\bar{g}_{i, j} \text { Map nocloud }
$$

where $\bar{g}_{i, j}$ is the logical inverse of $g_{i, j}$.

\section{EXPERIMENTS}

\section{A. Data Description}

In 2013, the Data Fusion Technical Committee of the IEEE Geoscience and Remote Sensing Society (GRSS) organized a contest involving two types data sources: a cloud-shadow hyperspectral image and a LiDAR derived digital surface model (DSM), both at the same spatial resolution $(2.5 \mathrm{~m})$ [20]. The competition was established to devise advanced methods for fusion and classification of HS and LiDAR data [21]. This data set was captured by the NSF-funded Center for Airborne Laser Mapping (NCALM) using the compact airborne spectrographic imager (CASI-1500) on June 2012 over the University of Houston campus and its neighboring urban area. The HS image has 144 spectral bands $(D=144)$ with a wavelength range from 380 to $1050 \mathrm{~nm}$. The whole scene of the data contains $349 \times 1905$ pixels. The ground truth provided for this data set contains 15 classes, summed up in Table II also mentioning between brackets the available numbers of training/test samples. The false color image and LiDAR image are shown in Fig. 1a and Fig. 1b, the distribution of training and test samples are shown in Fig. 1c and Fig. 1d The given scene contains a large cloud-shadow region (see 
Fig. 1a), which distorts the spectral radiance of objects in the HS image (darkening effect). More information can be found in [20].

\section{B. Experimental Setup}

The input HS image is transformed by PCA, and the first two $P C s$ are kept since they contain almost all of the variance in the data set (cumulative variation of more than 99\%). For the feature extraction (FE), we use a supervised method: nonparametric weighted feature extraction (NWFE) [34], as it has been shown to be efficient in many classification applications [32]. To generate the EMAPs, four attributes are considered: 1) (a) area $\lambda_{a}$ (related the size of the objects); 2) (s) standard deviation $\lambda_{s}$ (as a measure of homogeneity of the objects); 3 ) (d) diagonal of the box bounding the objects $\lambda_{d}$;4) (i) moment of inertia $\lambda_{i}$ (as a measure of the elongation of the objects). The values of each attribute are given as follows:

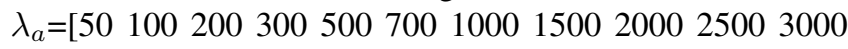
4000];

$\lambda_{s}=\left[\begin{array}{llllllllll}5 & 10 & 15 & 20 & 25 & 30 & 35 & 40 & 50 & 60\end{array}\right]$;

$\lambda_{d}=\left[\begin{array}{lllllllllll}5 & 10 & 25 & 50 & 75 & 100 & 150 & 200 & 300 & 400 & 500\end{array}\right] ;$

$\lambda_{i}=\left[\begin{array}{llllllllll}0.1 & 0.2 & 0.3 & 0.4 & 0.5 & 0.6 & 0.7 & 0.8 & 0.9 & 1\end{array}\right]$.

According to the number of selected values of each attribute, the dimension (denoted by $D_{1}$ ) of EMAPs ${ }_{h s i}$ is $D_{1}=134$, the dimension (denoted by $D_{2}$ ) of MAPs $\mathrm{Mid}_{l i d}$ is $D_{2}=67$.

The SVM classifier [39] with radial basis function (RBF) kernels is applied, containing two parameters: the penalty factor $C$ and the RBF kernel widths $\gamma . C$ is optimized within the given set $\left\{10^{-1}, 10^{0}, 10^{1}, 10^{2}, 10^{3}\right\}$ and $\gamma$ is optimized within the given set $\left\{10^{-3}, 10^{-2}, 10^{-1}, 10^{0}, 10^{1}\right\}$ by five-fold cross validation.

We compare our proposed framework with the following schemes: 1) using the original HS image $\left.\left(\operatorname{Raw}_{h s i}\right) ; 2\right)$ using spectral features Fspe extracted from the HS image by NWFE; 3) using spatial features Fspa extracted from EMAPs $_{h s i}$ by NWFE; 4) using elevation features Flid extracted from MAPs ${ }_{l i d}$ by NWFE; 5) using stacked features $\left(F E\right.$ stacked), stacking all spectral features, EMAPs ${ }_{h s i}$ and MAPs $_{l i d}$ first, similar as the approach of [18], and then extracting low-dimensional features by NWFE, as shown in Fig. 6b, 6) using fusion of stacked features Fspe, Fspa and Flid but only using the original training samples (Ffusion); this is the proposed approach without the co-training samples selection procedure. 7) using fused features from the generalized graphbased fusion method $(F g g f)$, the same as in the approach of [22].

The classification results are quantitatively evaluated by measuring the overall classification accuracy (OA), the average accuracy (AA), Kappa coefficient $(\kappa)$ on the test samples, shown in Fig. 1d.

\section{Effect of Number of Nearest Neighbors for Co-training Selection}

The number of nearest neighbors of each class center $(e)$ is an important parameter in the co-training samples selection procedure. On the one hand, when $e$ is too small, there will be insufficient co-training samples. On the other hand, large $e$ will lead to mislabeling of co-training samples, as some samples with different labels will be included in the nearest neighbors. To investigate the effect of the number of nearest neighbors on the classification accuracy, we performed classification experiments with different numbers of $e$. The number of nearest neighbors was changed from 50 to 250 with a stepsize of 10. Fig. 9 shows the OA for the whole scene and for the shadow area in function of an increasing number of nearest neighbors. As can be seen, the average OA increases as the number of nearest neighbors grows from 50 to 200, and then decreases with more nearest neighbors. This indicates that if $e$ is set to a small value, the selected number of co-training samples will be too small to allow to train the classifier; if $e$ is set to a large value, the possibility of mislabeling co-training samples increases, leading to poor classification performances. For this data set, we have set the number of nearest neighbors to 200 in our experiments.

\section{Classification Results on the data set}

This section mainly explores the efficiency of the proposed method, compared to the other methods. The resulting accuracies are reported in Tables III, III and IV] and the classification maps are shown in Fig. 10 for visual comparison. The best accuracy among different methods(in row) is highlighted in bold. From the tables and figures, we conclude the following:

1) The proposed framework improves all results in terms of the overall accuracy (OA), the average accuracy (AA), the Kappa coefficient $(\kappa)$ and the quality of the classification map. On the shadow-free region, it outperforms the state of the art by $2 \%$, in the cloudshadow region, the improvements are dramatic. On the whole scene, the proposed framework improves the OA with $3.87 \%-20.10 \%$ over the other schemes.

2) In general, it can be observed that fusion of multiple features (spectral, spatial and elevation features) leads to better classification performances in comparison with using one single type of features. This shows that the chosen sets of features are efficient and fusing them exploits the information contained in both data sources.

3) When investigating the classification accuracies for each class separately in Table IV] it can be clearly noticed that, when single features are used, the $\mathrm{Raw}_{h s i}$ approach produces better results on class 'Tree', whereas the Fspa scheme performs better on classes 'water', 'Residential' and 'Road'. However spectral or spatial features from the HS image perform poor on classes 'Commercial' and 'Railway'. On the contrary, Flid, extracted from the LiDAR image performs much better on these two classes. Classification accuracies for most classes improves by fusing those three features, especially for classes 'Residential', 'Road' and 'Parking Lot 2'. The generalized graph-based fusion method $(F g g f)$ [22] improves the classification accuracy on classes 'Grass Stressed', 'Tree' and 'Highway'. The proposed framework obtains the best classification accuracies on 9 of the 15 classes.

4) From the results reported on the shadow-free region (Table III) and the whole image (Table IV), one can 


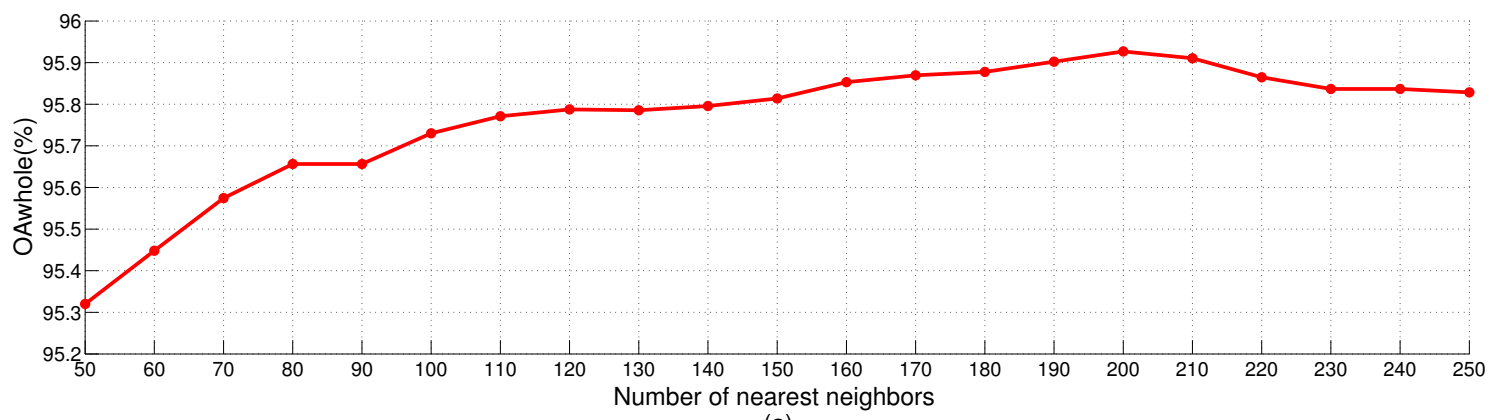

(a)

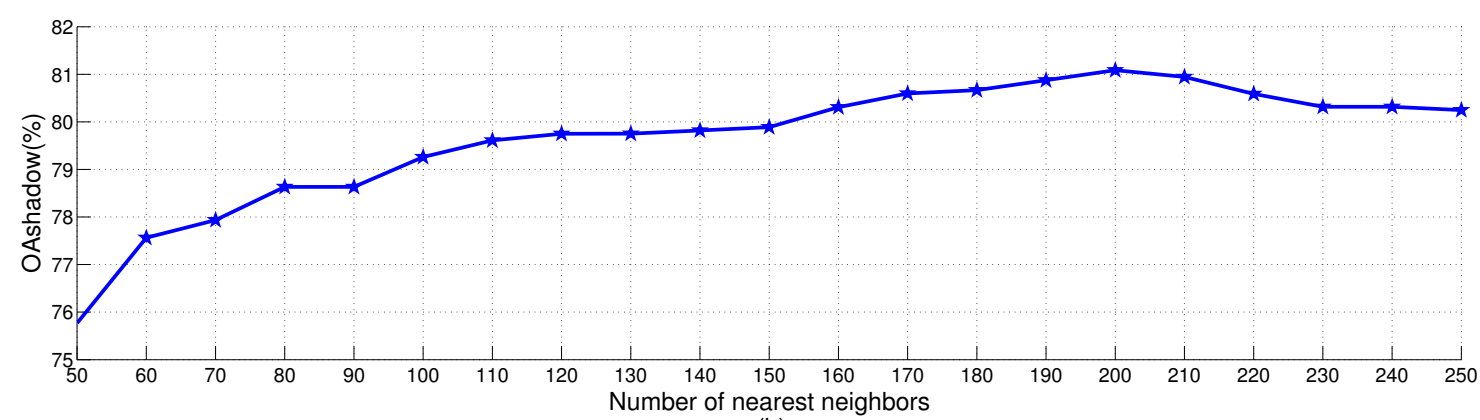

(b)

Fig. 9: (a) OA on the whole scene with increasing number of nearest neighbors; (b) OA on shadow area with increasing number of nearest neighbors.

TABLE II: Classification accuracies for the shadow-free regions obtained by the different methods.

\begin{tabular}{|l|c|c|c|c|c|c|c|c|}
\hline Features & Raw s $_{\text {s }}$ & Fspe & Fspa & Flid & FEstacked [18] & F fusion & Fggf $[22]$ & Proposed \\
\hline Number of Features & 144 & 15 & 15 & 15 & 45 & 45 & 22 & 45 \\
\hline Grass Healthy (198/875) & 98.86 & 98.40 & 99.54 & 69.71 & 99.66 & $\mathbf{1 0 0 . 0 0}$ & 99.77 & $\mathbf{1 0 0 . 0 0}$ \\
Grass Stressed (190/906) & 96.36 & 96.58 & 89.51 & 71.52 & 97.90 & 98.34 & $\mathbf{9 9 . 3 4}$ & 98.34 \\
Grass Synthetis (192/505) & 99.80 & $\mathbf{1 0 0}$ & $\mathbf{1 0 0}$ & 94.85 & $\mathbf{1 0 0}$ & $\mathbf{1 0 0}$ & $\mathbf{1 0 0}$ & $\mathbf{1 0 0}$ \\
Tree (188/986) & 98.49 & 95.54 & 89.55 & 74.54 & 98.99 & 97.26 & $\mathbf{9 9 . 1 9}$ & 97.26 \\
Soil (186/1056) & 97.92 & 98.58 & 98.30 & 83.14 & 99.43 & $\mathbf{9 9 . 8 3}$ & 99.81 & $\mathbf{9 9 . 8 3}$ \\
Water (182/143) & 95.10 & 95.10 & $\mathbf{9 9 . 3 0}$ & 84.62 & 95.80 & 95.80 & 95.80 & 95.80 \\
Residential (196/992) & 82.36 & 79.44 & 83.17 & 84.58 & 92.14 & $\mathbf{9 8 . 9 9}$ & 94.46 & $\mathbf{9 8 . 9 9}$ \\
Commercial (191/622) & 72.67 & 82.80 & 63.50 & 92.44 & 93.73 & $\mathbf{9 3 . 8 9}$ & 91.64 & $\mathbf{9 3 . 8 9}$ \\
Road (193/1040) & 79.35 & 76.66 & 80.50 & 66.28 & 86.74 & $\mathbf{9 4 . 4 3}$ & 89.34 & $\mathbf{9 4 . 4 3}$ \\
Highway (191/710) & 86.62 & 93.66 & 96.06 & 93.52 & 96.34 & $\mathbf{9 9 . 7 2}$ & 91.13 & $\mathbf{9 9 . 7 2}$ \\
Railway (181/902) & 91.57 & 90.47 & 87.58 & 96.78 & 96.78 & $\mathbf{9 9 . 2 2}$ & 95.01 & $\mathbf{9 9 . 2 2}$ \\
Parking Lot 1 (192/1041) & 84.34 & 78.19 & 88.18 & 66.38 & 89.15 & $\mathbf{9 7 . 9 8}$ & 81.08 & $\mathbf{9 7 . 9 8}$ \\
Parking Lot 2 (184/265) & 77.36 & 76.23 & 78.11 & 68.68 & 86.04 & $\mathbf{8 7 . 9 2}$ & 80.00 & $\mathbf{8 7 . 9 2}$ \\
Tennis Court (181/247) & 99.60 & 99.60 & $\mathbf{1 0 0}$ & 99.60 & $\mathbf{1 0 0}$ & $\mathbf{1 0 0}$ & $\mathbf{1 0 0}$ & $\mathbf{1 0 0}$ \\
Running Track (187/473) & 97.25 & 97.04 & $\mathbf{1 0 0}$ & 58.35 & 98.52 & 98.73 & 98.73 & 98.73 \\
\hline OA (\%) & 90.28 & 89.77 & 89.42 & 79.03 & 95.24 & $\mathbf{9 7 . 9 1}$ & 94.37 & $\mathbf{9 7 . 9 1}$ \\
AA (\%) & 90.58 & 90.55 & 90.22 & 80.33 & 95.37 & $\mathbf{9 7 . 4 7}$ & 94.35 & $\mathbf{9 7 . 4 7}$ \\
$\kappa$ & 0.894 & 0.889 & 0.885 & 0.773 & 0.948 & $\mathbf{0 . 9 7 7}$ & 0.939 & $\mathbf{0 . 9 7 7}$ \\
\hline
\end{tabular}

TABLE III: Classification accuracies for the cloud-shadow regions obtained by the different methods.

\begin{tabular}{|l|c|c|c|c|c|c|c|c|}
\hline Features & Raw $h$ si & Fspe & Fspa & Flid & FEstacked $[18$ & Ffusion & Fggf $[22]$ & Proposed \\
\hline Number of Features & 144 & 15 & 15 & 15 & 45 & 45 & 22 & 45 \\
\hline Grass Healthy (85/178) & 0.00 & 0.00 & 0.00 & 55.06 & 0.00 & 0.00 & 0.00 & $\mathbf{8 4 . 2 6}$ \\
Grass Stressed (102/158) & 0.00 & 0.00 & 0.00 & 51.89 & 0.00 & 0.00 & $\mathbf{9 1 . 7 8}$ & 89.24 \\
Tree (89/70) & 0.00 & 0.00 & 0.00 & $\mathbf{1 0 0 . 0 0}$ & 0.00 & 0.00 & $\mathbf{1 0 0 . 0 0}$ & $\mathbf{1 0 0 . 0 0}$ \\
Residential (75/80) & 1.25 & 71.25 & $\mathbf{9 0 . 0 0}$ & 58.75 & 0.00 & 66.25 & 63.74 & 77.50 \\
Commercial (121/431) & 29.23 & 0.00 & 0.00 & 82.60 & 69.37 & 49.19 & 84.74 & $\mathbf{8 8 . 1 7}$ \\
Road (71/19) & 0.00 & 0.00 & 0.00 & $\mathbf{6 3 . 1 6}$ & 0.00 & 0.00 & 52.63 & 57.89 \\
Highway (92/326) & 0.00 & 51.23 & 0.00 & 46.63 & 15.34 & 23.93 & $\mathbf{7 6 . 0 7}$ & 69.49 \\
Railway (111/152) & 4.61 & 71.05 & 15.79 & 92.11 & 91.45 & 92.11 & $\mathbf{9 3 . 4 2}$ & 92.76 \\
Parking Lot 2 (27/20) & 5.00 & $\mathbf{1 0 . 0 0}$ & 0.00 & 5.00 & 0.00 & 0.00 & 0.00 & $\mathbf{1 0 . 0 0}$ \\
\hline OA (\%) & 9.42 & 22.31 & 6.70 & 66.71 & 34.96 & 34.54 & 71.97 & $\mathbf{8 1 . 1 5}$ \\
AA (\%) & 4.45 & 22.61 & 11.75 & 61.56 & 20.52 & 36.60 & 62.49 & $\mathbf{7 4 . 3 7}$ \\
$\kappa$ & 0.160 & 0.134 & 0.111 & 0.679 & 0.206 & 0.230 & 0.717 & $\mathbf{0 . 7 9 6}$ \\
\hline
\end{tabular}


TABLE IV: Classification accuracies for the whole image obtained by the different methods.

\begin{tabular}{|l|c|c|c|c|c|c|c|c|}
\hline Features & Raw $h$ si & Fspe & Fspa & Flid & FEstacked [18] & Ffusion & Fggf [22] & Proposed \\
\hline Number of Features & 144 & 15 & 15 & 15 & 45 & 45 & 22 & 45 \\
\hline Grass Healthy (1053) & 82.15 & 81.77 & 82.72 & 57.93 & 82.81 & 83.00 & 82.91 & $\mathbf{9 7 . 3 4}$ \\
Grass Stressed (1064) & 82.05 & 82.24 & 76.22 & 60.90 & 83.36 & 83.74 & $\mathbf{9 9 . 4 4}$ & 96.99 \\
Grass Synthetis (505) & 99.80 & $\mathbf{9 9 . 8 0}$ & $\mathbf{1 0 0}$ & 94.85 & $\mathbf{1 0 0}$ & $\mathbf{1 0 0}$ & $\mathbf{1 0 0}$ & $\mathbf{1 0 0}$ \\
Tree (1056) & 92.90 & 89.20 & 83.62 & 76.23 & 92.42 & 91.48 & $\mathbf{9 9 . 2 4}$ & 97.44 \\
Soil (1056) & 97.92 & 98.58 & 98.30 & 83.14 & 99.43 & 99.81 & 99.81 & $\mathbf{9 9 . 8 3}$ \\
Water (143) & 95.10 & 95.10 & $\mathbf{9 9 . 3 0}$ & 84.62 & 95.80 & 95.80 & 95.80 & 95.80 \\
Residential (1072) & 76.31 & 78.82 & 83.68 & 82.65 & 85.26 & 97.11 & 91.42 & $\mathbf{9 7 . 3 8}$ \\
Commercial (1053) & 54.89 & 48.91 & 37.51 & $\mathbf{9 2 . 9 7}$ & 83.76 & 75.59 & 92.50 & 91.55 \\
Road (1059) & 78.00 & 75.35 & 79.13 & 66.48 & 85.27 & 92.82 & 88.76 & $\mathbf{9 3 . 8 6}$ \\
Highway (1036) & 59.36 & 80.31 & 65.83 & 70.17 & 70.85 & 75.87 & 84.85 & $\mathbf{9 0 . 1 5}$ \\
Gailway (1054) & 79.03 & 87.67 & 77.23 & 96.39 & 97.25 & $\mathbf{9 9 . 1 5}$ & 95.73 & 98.58 \\
Parking Lot 1 (1041) & 84.34 & 78.19 & 88.18 & 66.38 & 89.15 & 97.69 & 81.08 & $\mathbf{9 7 . 6 9}$ \\
Parking Lot 2 (285) & 72.28 & 71.58 & 72.63 & 63.86 & 80.00 & 77.89 & 74.39 & $\mathbf{8 2 . 4 6}$ \\
Tennis Court (247) & 99.60 & 99.60 & $\mathbf{1 0 0}$ & 99.60 & $\mathbf{1 0 0}$ & $\mathbf{1 0 0}$ & $\mathbf{1 0 0}$ & $\mathbf{1 0 0}$ \\
Running Track (473) & 97.25 & 97.04 & $\mathbf{1 0 0}$ & 58.35 & 98.52 & 98.73 & 98.73 & 98.73 \\
\hline OA (\%) & 80.78 & 81.96 & 79.70 & 75.82 & 88.16 & 90.44 & 92.05 & $\mathbf{9 5 . 9 2}$ \\
AA (\%) & 83.40 & 84.29 & 82.96 & 76.97 & 89.55 & 91.25 & 92.31 & $\mathbf{9 5 . 6 5}$ \\
$\kappa$ & 0.793 & 0.805 & 0.779 & 0.738 & 0.871 & 0.896 & 0.914 & $\mathbf{0 . 9 5 8}$ \\
\hline
\end{tabular}

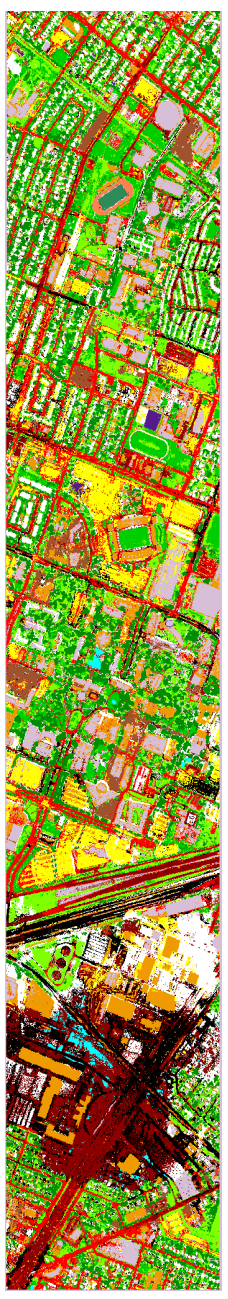

(a)

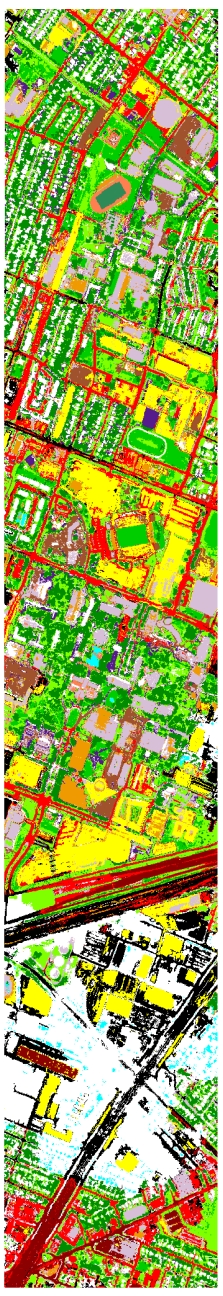

(b)

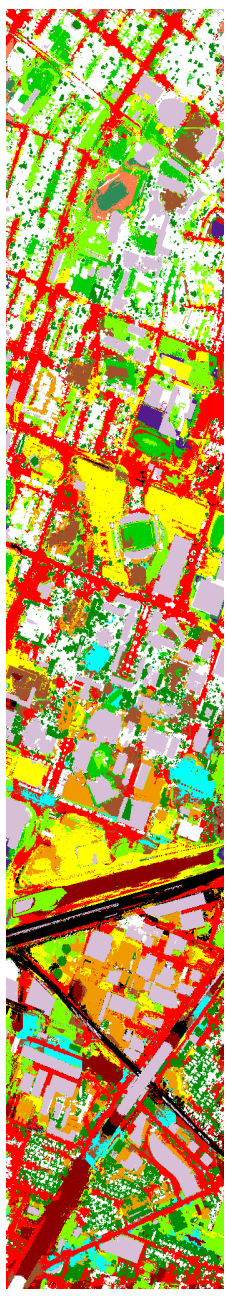

(c)

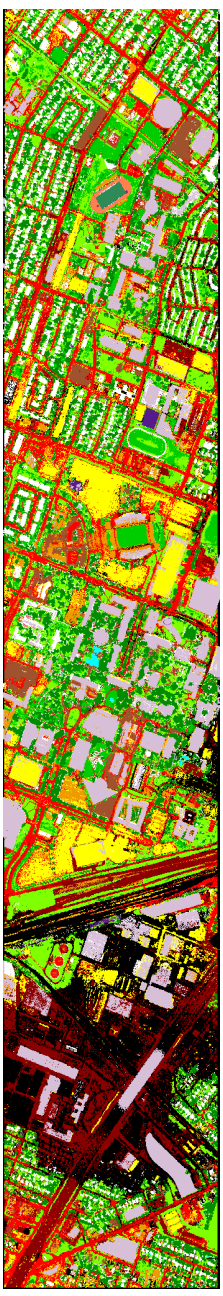

(d)

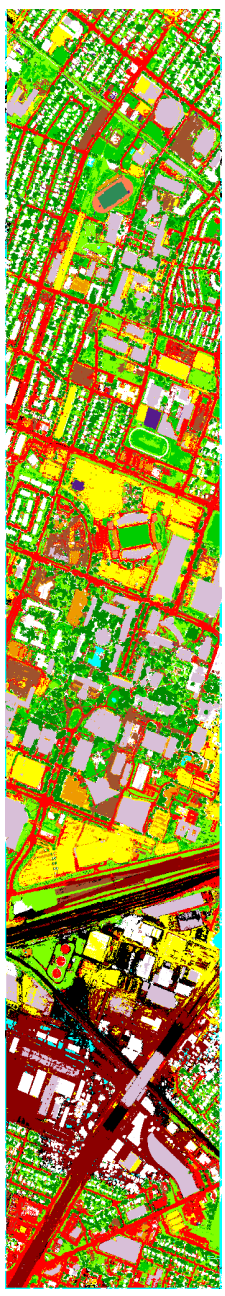

(e)

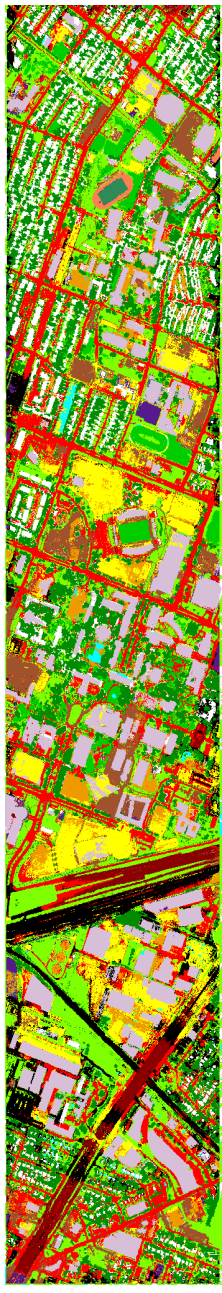

(f)

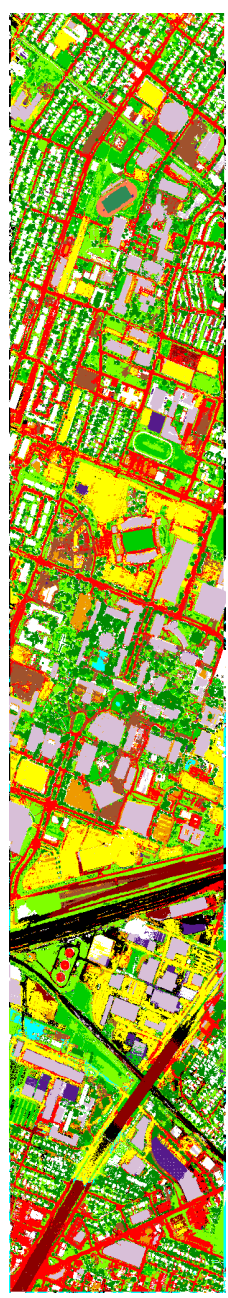

(g)

Fig. 10: Classification maps produced by the different methods. (a) Fspe; (b) Fspa; (c) Flid; (d) FEstacked; (e) Ffusion; (f) Fggf; (g) Proposed.

infer that fusing the features extracted from each source (HS image, EMAPs L $_{h i}$ and MAPs $s_{l i d}$ ) works better than using the features extracted from the stacked vector of the original HS image, EMAPs ${ }_{h s i}$ and $\mathrm{MAPs}_{l i d}$, with an improvement of almost 3\%. The main reason behind this is that, because of their different nature, when fusing features from different sources and then projecting them on a lower dimensional space, information gets mixed 
up and lost.

5) By comparing the classification maps in Fig. 10 and classification accuracies on the cloud-shadow region (Table [III), we can see that most of the objects under the cloud-shadow region are not well classified when only using the training samples located in the shadowfree region. Some objects in the cloud-shadow region are classified better by using features extracted from LiDAR image, because the elevation information contained in the morphological features of LiDAR image is not influenced by the cloud. But for many other objects, the results are not good as the elevation information is not sufficiently discriminative. Taking all feature sources into consideration does not much improve the classification accuracy for most of the classes. The proposed framework leads to an improved classification of most classes, due to the selection and use of specific training samples in the cloud-shadow region.

Moreover, as the described data set [20] is very popular and open access, it has been used in many recently state of the arts, such as in [18], [22], [23] and [24]. Compared with the experimental results from these references, the proposed scheme performs better on either cloud-shadow or shadowfree regions, with overall classification accuracy $97.91 \%$ and $81.15 \%$ respectively. Thus proves the proposed fused features are effective and distinguishable, and selecting new training samples from the cloud-shadow regions is an efficient solution.

\section{CONCLUSION}

In this paper, we developed a new method for classification of cloud mixed remote sensing scenes by fusion of hyperspectral and LiDAR data. The proposed method selects new samples as co-training samples in the cloud-shadow rgions and classifies shadow-free and cloud-shadow regions separately using their own sets of training samples. In order to better combine HS and LiDAR data, additional spatial and elevation features are extracted from HS and LiDAR data, and effectively integrated without any regularization or weight parameters. Experimental results on the classification of the real cloud-shadow HS and LiDAR data show the efficiency of the proposed framework. In addition, the proposed approach can be thought of as a general framework, in which the feature extraction step can be replaced by any other technique (kernel PCA, supervised or semi-supervised feature extraction, ...), possibly to improve classification accuracies. Moreover, the proposed framework is completely open and flexible in its capacity to integrate more features.

Recent Earth observation missions (Landsat series from NASA, Sentinel series from ESA) boost the use of the multisensor remote sensing imagery. However, cloud/shadow effects cannot be avoided in the optical sensors. Other sensors (e.g., synthetic aperture radar, thermal infrared, LiDAR, etc.) can provide complementary information for these cloud/shadow regions. The proposed framework is applicable for fusion of optical hyperspectral images and other images (e.g., SAR, thermal infrared), where multi-sensor images are available. On the other hand, de-shadowing is typically used as a preprocessing step before applications. Fusion of multi-sensor data for de-shadowing (e.g., hyperspectral image restoration) will be our future work.

\section{ACKNOWLEDGMENT}

The authors would like to thank the Hyperspectral Image Analysis group and the NSF Funded Center for Airborne Laser Mapping (NCALM) at the University of Houston for providing the data sets used in this study. Wenzhi Liao is a postdoctoral fellow of the Research Foundation Flanders (FWO-Vlaanderen) and acknowledges its support.

\section{REFERENCES}

[1] D. Bruce, A. Lawrence, F. Ross, M. Elizabeth, C. Douglas, T. Joel, G. Jeffrey, J. Kenneth, Vuong Ly and M. Paul, "NASA Goddard's LiDAR, hyperspectral and thermal (G-LiHT) airborne imager," Remote Sens., vol. 5, no. 8, pp. 4045-4066, Aug. 2013.

[2] H. Zhang, H. Zhai, L. Zhang, P. Li, "Spectral-spatial sparse subspace clustering for hyperspectral remote sensing images," IEEE Trans. Geosci. Remote Sens. vol. 54, no. 6, pp. 3672-3684, June 2016.

[3] R. Luo, W. Liao, X. Huang, Y. Pi, W. Philips. "Feature extraction of hyperspectral images with semi-supervised graph learning," IEEE J. Sel. Top. Appl. Earth Observat. Remote Sens. vol. 9, no. 9, pp. 4389-4399, Sep. 2016.

[4] H. Zhang, J. Li , Y. Huang, L. Zhang, "A nonlocal weighted joint sparse representation classification method for hyperspectral imagery," IEEE J. Sel. Top. Appl. Earth Observat. Remote Sens. vol. 7, no. 6, pp. 2056-2065, Jun. 2014.

[5] J. Jung, E. Pasolli, S. Prasad, J. Tilton and M. Crawford, "A framework for land cover classification using discrete return LiDAR data: Adopting pseudo-waveform and hierarchical segmentation," IEEE J. Sel. Topics Appl. Earth Observ. Remote Sens., vol. 7, no. 2, pp. 491-502, Feb. 2014

[6] Y. Gu, Q. Wang, X. Jia and J. A. Benediktsson, "A novel MKL model of integrating LiDAR data and MSI for urban area classification," IEEE Trans. Geosci. Remote Sens., vol. 53, no. 4, pp. 5312-5326, Oct. 2015.

[7] A. F. Elakshe, "Fusion of hyperspectral images and lidar-based DEMs for coastal mapping," Optics Lasers Eng., vol. 46, no. 7, pp. 493-498, Jul. 2008.

[8] M. Dalponte, L. Bruzzone and D. Gianelle, "Fusion of hyperspectral and LiDAR remote sensing data for classification of complex forest areas," IEEE Trans. Geosci. Remote Sens., vol. 46, no. 5, pp. 1416-1427, May 2008.

[9] N. Yokoya, S. Nakazawa, T. Matsuki and A. Iwasaki, "Fusion of hyperspectral and LiDAR data for landscape visual quality assessment," IEEE J. Sel. Top. Appl. Earth Observat. Remote Sens., vol. 7, no. 6, pp. 2419 2425, Jun. 2014.

[10] L. Naidooa, M. Choa, R. Mathieua and G. Asner, "Classification of savanna tree species, in the greater kruger national park region, by integrating hyperspectral and lidar data in a random forest data mining environment," ISPRS J. Photogramm. Remote Sens., vol. 69, pp. 167-179, Apr. 2012.

[11] M. Shimoni, G. Tolt, C. Perneel and J. Ahlberg, "Detection of vehicles in shadow areas," In Proc. 3rd Workshop Hyperspectral Image Signal Process.: Evol. Remote Sens. (WHISPERS), pp. 14, 2011.

[12] M. Pedergnana, P. Reddy Marpu, M. Dalla Mura, J. A. Benediktsson and L. Bruzzone, "Classification of remote sensing optical and LiDAR data using extended attribute profiles," IEEE Journals on Selected Topics in Signal Processing, vol. 6, no. 7, pp. 856-865, Nov. 2012.

[13] W. Liao, R. Bellens, A. Pižurica, S. Gautama and W. Philips, "Combining feature fusion and decision fusion for classification of Hyperspectral and LiDAR Data," In Proc. IEEE IGARSS, Quebec City, QC , Jul. 13-18 , vol. 1, pp. 1241-1244, Jul. 2014.

[14] C. Chen, W. Li, H. Su and K. Liu, "Spectral-spatial classification of hyperspectral image based on kernel extreme learning machine," Remote Sens., vol. 6, no. 6, pp. 5795-5814, Jun. 2014.

[15] P. Ghamisi, J. Benediktsson and M. Ulfarsson, "Spectral-spatial classification of hyperspectral images based on hidden Markov random fields," IEEE Trans. Geosci. and Remote Sens., vol. 52, no. 5, pp. 2565-2574, May 2014.

[16] W. Liao, R. Bellens, A. Pižurica, W. Philips and Y. Pi, "Classification of hyperspectral data over urban areas using directional morphological profiles and semi-supervised feature extraction," IEEE J. Sel. Topics Appl. Earth Observ. Remote Sens., vol. 5, no. 4, pp. 1177-1190, Aug. 2012. 
[17] M. Dalla Mura, J. A. Benediktsson, B. Waske and L. Bruzzone, "Extended profiles with morphological attribute filters for the analysis of hyperspectral data," Int. J. Remote Sens., vol. 31, no. 22, pp. 59755991, Nov. 2010.

[18] M.Khodadadzadeh ; J. Li; S. Prasad and A. Plaza, "Fusion of hyperspectral and LiDAR remote sensing data using multiple feature learning," IEEE J. Sel. Topics Appl. Earth Observ. Remote Sens., vol. 8, no. 6, pp. 2971-2983, Jun. 2015.

[19] G. F. Hughes, "On the mean accuracy of statistical pattern recognizers," IEEE Transactions on Information Theory, vol.14, no.1, pp. 55-63, 1968.

[20] 2013 IEEE GRSS Data Fusion Contest, Online: http://www.grssieee.org/community/technical-committees/data-fusion/.

[21] C. Debes, et al, "Hyperspectral and LiDAR data fusion: outcome of the 2013 GRSS data fusion contest," IEEE J. Sel. Topics Appl. Earth Observ. Remote Sens., vol. 7, no. 6, pp. 2405-2418, Jun. 2014.

[22] W. Liao, A. Pižurica, R. Bellens, S. Gautama and W. Philips, "Generalized graph-based fusion of hyperspectral and LiDAR data using morphological features," IEEE Geosci. Remote Sens. Lett., vol. 12, no. 3, pp. 552-556, Mar. 2015.

[23] R. Bao, J. Xia, M. Dalla Mura, P. Du, J. Chanussot, J. Ren, “Combining morphological attribute profiles via an ensemble method for hyperspectral image classification," IEEE Geosci. Remote Sens. Lett., vol. 13, no. 3, pp. 359-363, Mar. 2016.

[24] Z. Zhong, B. Fan, K. Ding, H. Li, S. Xiang, C. Pan, "Efficient multiple feature fusion with hashing for hyperspectral imagery classification: a comparative study," IEEE Trans. Geosci. and Remote Sens., vol. 54, no. 8, pp. 4461-4478, Aug. 2016.

[25] P. Ghamisi, B. Hofle, X. Zhu, "Hyperspectral and LiDAR data fusion using extinction profiles and deep convolutional neural network," IEEE J. Sel. Topics Appl. Earth Observ. Remote Sens., to be published. doi: 10.1109/JSTARS.2016.2634863.

[26] Z. Zhu and C. E. Woodcock, "cloud, cloud shadow, and snow detection in multitemporal landsat data: An algorithm designed specifically for monitoring land cover change," Remote Sens. Environ., vol. 152, pp. $217-$ 234, Sep. 2014

[27] M. Dalla Mura, J. Benediktsson, B. Waske and L. Bruzzone, "Morphological attribute profiles for the analysis of very high resolution images," IEEE Trans. Geosci. Remote Sens., vol. 48, no. 10, pp. 3747-3762, Oct. 2010.

[28] D. Tuia, C. Persello, L. Bruzzone, "Domain adaptation for the classification of remote sensing data: An overview of recent advances," IEEE Geosci. Remote Sens. Mag., vol. 4, no. 2, pp. 41-57, Jun. 2016.

[29] M. Fauvel, J. A. Benediktsson, J. Chanussot and J. R. Sveinsson, "Spectral and spatial classification of hyperspectral data using SVMs and morphological profile," IEEE Trans. Geosci. Remote Sens., vol. 46, no. 11, pp. 3804-3814, Nov. 2008.

[30] M. Pesaresi and J. A. Benediktsson, "A new approach for the morphological segmentation of high-resolution satellite imagery," IEEE Trans. Geosci. Remote Sens., vol. 39, no. 2, pp. 309-320, Feb. 2001.

[31] M. Dalla Mura, A. Villa, J. A. Benediktsson, J. Chanussot and L. Bruzzone, "Classification of hyperspectral images by using extended morphological attribute profiles and independent component analysis," IEEE Geosci. Remote Sens. Lett., vol. 8, no. 3, pp. 541-545, May 2011.

[32] P. Ghamisi, J. Benediktsson and J. Sveinsson, "Automatic spectralspatial classification framework based on attribute profiles and supervised feature extraction," IEEE Trans. Geosci. and Remote Sens., vol. 52, no. 9, pp. 5771-5782, Sep. 2014.

[33] J. A. Benediktsson, J. A. Palmason and J. R. Sveinsson, "Classification of hyperspectral data from urban areas based on extended morphological profiles," IEEE Trans. Geosci. Remote Sens., vol. 40, no. 3, pp. 480-491, Mar. 2005.

[34] B. Kuo and D. Landgrebe, "Nonparametric weighted feature extraction for classification," IEEE Trans. Geosci. Remote Sens., vol. 42, no. 5, pp. 1096-1105, May 2004.

[35] T. Arvidson, J. Gasch and S. N. Goward, "Landsat-7's long-term acquisition plan-An innovative approach to building a global imagery archive," Remote Sens. Environ., vol. 78. no. 1-2, pp. 13-26, Oct. 2001.

[36] Y. Luo, A. P. Trishchenko and K. V. Khlopenkov, "Developing clearsky, cloud and cloud shadow mask for producing clear-sky composites at 250-meter spatial resolution for the seven MODIS land bands over Canada and North America." Remote Sens. Environ., vol. 112, no. 12, pp. 4167-4185, Dec. 2008.

[37] Z. Zhu and C. E. Woodcock, "Object-based cloud and cloud shadow detection in Landsat imagery," Remote Sens. Environ., vol. 118, no. 15, pp. 83-94, Mar. 2012.

[38] D. Frantz, A. Roder, T. Udelhoven and M. Schmidt, "Enhancing the detectability of clouds and their shadows in multitemporal dryland
Landsat imagery: extending fmask," IEEE Geosci. Remote Sens. Lett., vol. 12 , no. 6, pp. 1242-1246, Jun. 2015.

[39] C. Chang and C. Lin, "LIBSVM-A library for support vector machines," [Online], Available: https://www.csie.ntu.edu.tw/ cjlin/libsvm/, Dec. 2016.

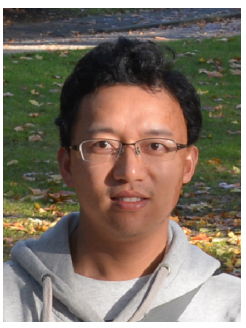

Renbo Luo (S'16) received the B.S. degree in Automation Control Engineering from South China University of Technology, Guangzhou, China, in 2010. Currently, he is working toward the Ph.D. degree in Control Theory and Control Engineering from South China University of Technology, China, and the Ph.D. Degree in Computer Science Engineering from Ghent University, Belgium.

His research interests include image processing, object recognition, data fusion, domain adaptation and remote sensing applications

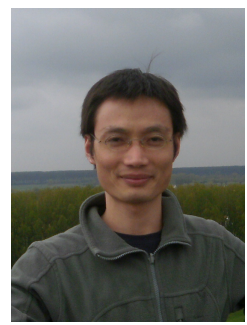

Wenzhi Liao (S'10-M'14-SM'16) received the B.S degree in mathematics from Hainan Normal University, HaiKou, China, in 2006, the PhD Degree in Engineering from South China University of Technology (China) in 2012 and the PhD Degree in Computer Science Engineering from Ghent University (Belgium) in 2012.

Since 2012 he works as a postdoc at Ghent University, he is a Postdoctoral Fellow with the Fund for Scientific Research in Flanders-FWO. His current research interests include pattern recognition, remote sensing, and image processing. In particular, his interests include mathematical morphology, multi-task feature learning, multi-sensor data fusion, hyperspectral image restoration.

He is a member of the Geoscience and Remote Sensing Society (GRSS) and IEEE GRSS Data Fusion Technical Committee (DFTC). He was the recipient of the "Best Paper Challenge" Awards on both 2013 IEEE GRSS Data Fusion Contest and 2014 IEEE GRSS Data Fusion Contest.

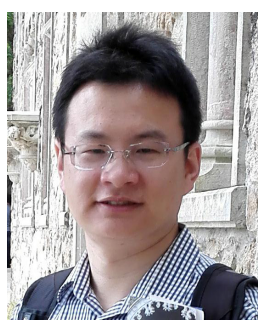

Hongyan Zhang (M'13-SM'16) received the B.S. degree in geographic information system and the $\mathrm{Ph} . \mathrm{D}$. degree in photogrammetry and remote sensing from Wuhan University, China, in 2005 and 2010, respectively.

He has been currently an Associate Professor with the State Key Laboratory of Information Engineering in Surveying, Mapping, and Remote Sensing, Wuhan University since 2013. He has authored/co-authored more than 60 research papers His research interests include image reconstruction for quality improvement, hyperspectral image processing, sparse representation and low rank methods for sensing image imagery.

Dr. Zhang is a Reviewer of more than 20 international academic journals, including IEEE Transaction on Geoscience and remote sensing, IEEE Transaction on Image processing, IEEE Journal of Selected Topics in Applied Earth Observations and Remote Sensing, IEEE Geoscience and remote sensing Letters and so on. 


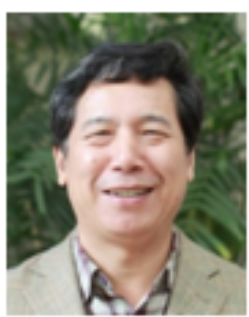

Liangpei Zhang (M'06-SM'08) received the B.S. degree in physics from Hunan Normal University, ChangSha, China, in 1982, the M.S. degree in optics from the Xi'an Institute of Optics and Precision Mechanics of Chinese Academy of Sciences, Xi'an, China, in 1988, and the Ph.D. degree in Photogrammetry and Remote Sensing from Wuhan University, Wuhan, China, in 1998.

$\mathrm{He}$ is currently the Head of the Remote Sensing Division, State Key Laboratory of Information Engineering in Surveying, Mapping and Remote Sensing, Wuhan University. He is also a Chang-Jiang Scholar Chair Professor appointed by the Ministry of Education of China. He is currently a Principal Scientist for the China State Key Basic Research Project (20112016) appointed by the Ministry of National Science and Technology of China to lead the remote sensing program in China. He has more than 500 research papers and five books. He is the holder of 15 patents. He has won the 2010 the best paper Boeing award and 2013 best paper ERDAS award of American Society of Photogrammetry and Remote Sensing (ASPRS), respectively His research interests include hyperspectral remote sensing, high-resolution remote sensing, image processing, and artificial intelligence.

Dr. Zhang is the Founding Chair of the IEEE Geoscience and Remote Sensing Society (GRSS) Wuhan Chapter. He is a Fellow of the Institution of Engineering and Technology, an Executive Member (Board of Governors) of the China National Committee of the International GeosphereBiosphere Programme, an Executive Member of the China Society of Image and Graphics,etc. He regularly serves as a Cochair of the series International Society for Optics and Photonics (SPIE) Conferences on Multispectral Image Processing and Pattern Recognition, the Conference on Asia Remote Sensing, and many other conferences. He edits several conference proceedings, issues, and geoinformatics symposiums. He also serves as an Associate Editor of the International Journal of Ambient Computing and Intelligence, the International Journal of Image and Graphics, the International Journal of Digital Multimedia Broadcasting, the Journal of Geo-spatial Information Science, the Journal of Remote Sensing, and the IEEE TRANSACTIONS ON GEOSCIENCE AND REMOTE SENSING; he is a Guest Editor of the Journal of Applied Remote Sensing and the Journal of Sensors. He was the General Chair for the Fourth IEEE GRSS Workshop on Hyperspectral Image and Signal Processing: Evolution in Remote Sensing (WHISPERS) and a Guest Editor of the IEEE Journal of Selected Topics in Applied Earth Observations and Remote Sensing (JSTARS). He was the recipient of the Best Reviewer Awards from the IEEE GRSS for his service to the IEEE JSTARS in 2012 and the IEEE Geoscience and Remote Sensing Letters in 2014. He was also the recipient of the 2010 Best Paper Boeing Award and the 2013 Best Paper ERDAS Award from the American Society of Photogrammetry and Remote Sensing. His research teams were the recipient of the top three prizes of the IEEE GRSS 2014 Data Fusion Contest, and his students have been selected as the winners or finalists of the IEEE International Geoscience and Remote Sensing Symposium Student Paper Contest in recent years.

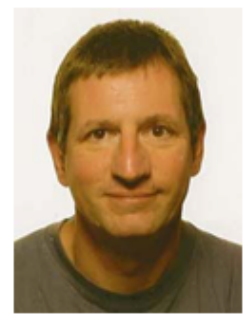

Paul Scheunders (M'98-SM'14) received the B.S. degree and the Ph.D. degree in physics, with work in the field of statistical mechanics, from the University of Antwerp, Antwerp, Belgium, in 1983 and 1990, respectively. In 1991, he became a research associate with the Vision Lab, Department of Physics, University of Antwerp, where he is currently a professor. His current research interest includes remote sensing and hyperspectral image processing. He has published over 200 papers in international journals and proceedings in the field of image processing, pattern recognition and remote sensing.

Paul Scheunders is Associate Editor of the IEEE Transactions in Geoscience and Remote Sensing, and has served as program committee member in numerous international conferences on remote sensing. He is senior member of the IEEE Geoscience and Remote Sensing Society.

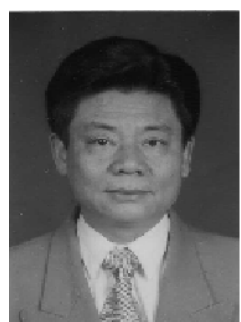

Youguo Pi received the Diploma degree in automation engineering from Chongqing University China and the ph.D degree in Mechanical engineering from South China University of Technology, in 1982 and 1998 ,respectively.

From July 1998 to June 2002, he was with the Information Technology Department, Automation Engineering Center, Academy Guangdong Province. Since July 2002, He has been with the College of Automation Science and Engineering, South China University of Technology, where he is currently a full-time Professor. Some of the recent research activities in the group including image processing and patter recognition and motion control. Important application areas targeted by the group include Intelligent Chinese character formation and servo system.

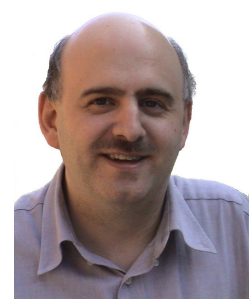

Wilfried Philips (S'90-M'93-SM'10) was born in Aalst, Belgium, on October 19, 1966. In 1989, he received the Diploma degree in electrical engineering and in 1993 the Ph.D. degree in applied sciences, both from Ghent University, Belgium.

Since November 1997 he is a lecturer at the Department of Telecommunications and Information Processing of Ghent University. Currently he is senior full professor at the Department of $\mathrm{T}$ elecommunications and Information Processing of Ghent University, where he heads the research group "Image Processing and Interpretation." His main research interests are in the domains of image and video restoration and multi-camera computer vision. 